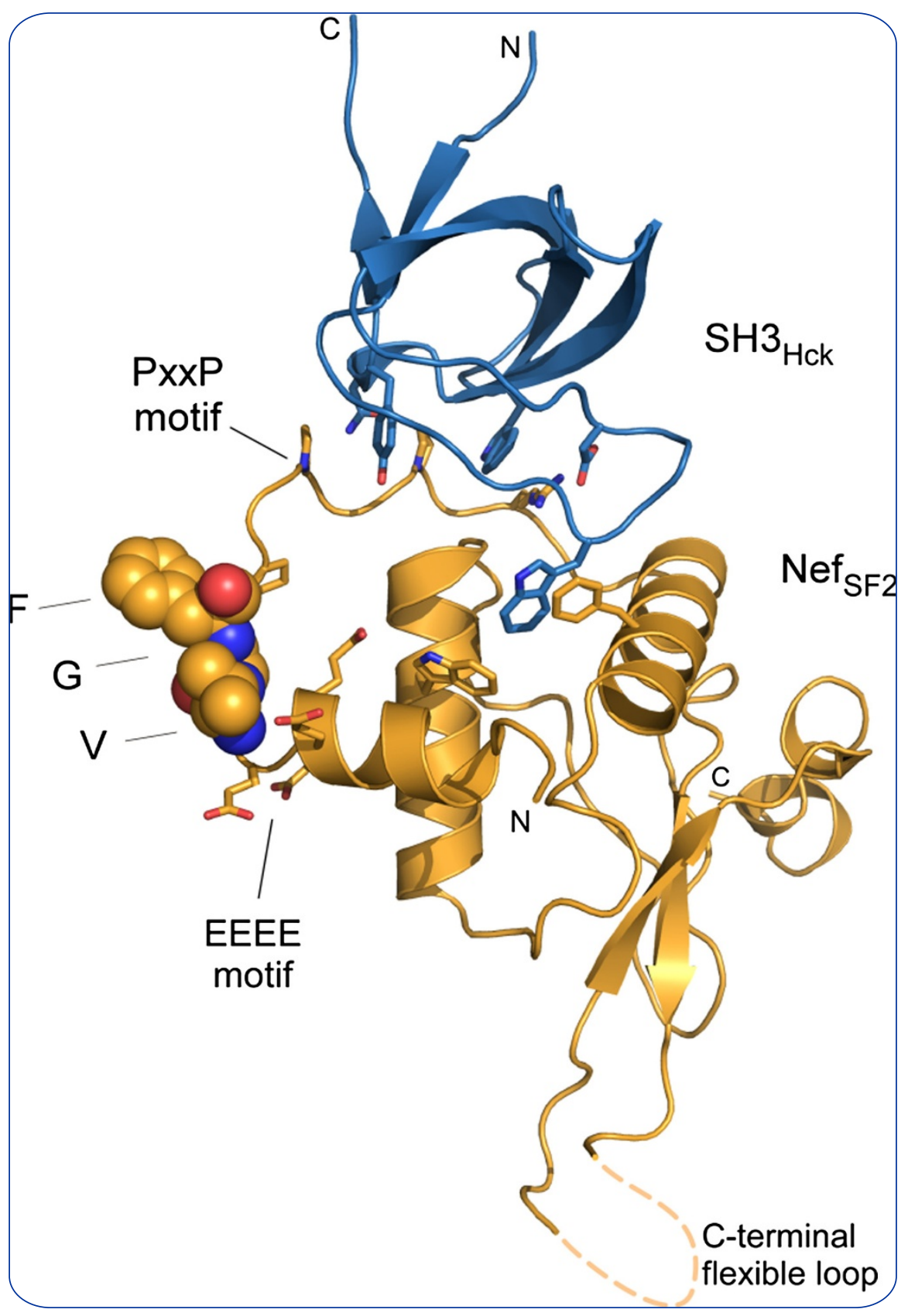

Identification of a highly conserved valine-glycinephenylalanine amino acid triplet required for HIV-1 Nef function

Meuwissen et al. 


\title{
Identification of a highly conserved valine-glycine- phenylalanine amino acid triplet required for HIV-1 Nef function
}

Pieter J Meuwissen ${ }^{1}$, Bettina Stolp², Veronica Iannucci ${ }^{1}$, Jolien Vermeire ${ }^{1}$, Evelien Naessens ${ }^{1}$, Kalle Saksela ${ }^{3}$, Matthias Geyer ${ }^{4}$, Guido Vanham ${ }^{5}$, Kevin K Arien ${ }^{1,6}$, Oliver T Fackler ${ }^{2}$ and Bruno Verhasselt ${ }^{1 *}$

\begin{abstract}
Background: The Nef protein of HIV facilitates virus replication and disease progression in infected patients. This role as pathogenesis factor depends on several genetically separable Nef functions that are mediated by interactions of highly conserved protein-protein interaction motifs with different host cell proteins. By studying the functionality of a series of nef alleles from clinical isolates, we identified a dysfunctional HIV group O Nef in which a highly conserved valine-glycine-phenylalanine (VGF) region, which links a preceding acidic cluster with the following proline-rich motif into an amphipathic surface was deleted. In this study, we aimed to study the functional importance of this VGF region.

Results: The dysfunctional HIV group $\mathrm{O} 8$ nef allele was restored to the consensus sequence, and mutants of canonical (NL4.3, NA-7, SF2) and non-canonical (B2 and C1422) HIV-1 group M nef alleles were generated in which the amino acids of the VGF region were changed into alanines (VGF $\rightarrow A A A)$ and tested for their capacity to interfere with surface receptor trafficking, signal transduction and enhancement of viral replication and infectivity. We found the VGF motif, and each individual amino acid of this motif, to be critical for downregulation of MHC-I and CXCR4. Moreover, Nef's association with the cellular p21-activated kinase 2 (PAK2), the resulting deregulation of cofilin and inhibition of host cell actin remodeling, and targeting of Lck kinase to the trans-golgi-network (TGN) were affected as well. Of particular interest, VGF integrity was essential for Nef-mediated enhancement of HIV virion infectivity and HIV replication in peripheral blood lymphocytes. For targeting of Lck kinase to the TGN and viral infectivity, especially the phenylalanine of the triplet was essential. At the molecular level, the VGF motif was required for the physical interaction of the adjacent proline-rich motif with Hck.
\end{abstract}

Conclusion: Based on these findings, we propose that this highly conserved three amino acid VGF motif together with the acidic cluster and the proline-rich motif form a previously unrecognized amphipathic surface on Nef. This surface appears to be essential for the majority of Nef functions and thus represents a prime target for the pharmacological inhibition of Nef.

Keywords: HIV, Nef, Sequence motifs, SH3 domain binding, Cytoskeleton, Lck, Receptor downregulation, Infectivity, Replication

\footnotetext{
* Correspondence: Bruno.Verhasselt@ugent.be

'Department of Clinical Chemistry, Microbiology, and Immunology, Ghent

University, Ghent, (B-9000), Belgium

Full list of author information is available at the end of the article
} 


\section{Background}

Primate immunodeficiency virus Nef is a $25-35 \mathrm{kDa}$ protein expressed by a conserved open reading frame, which partially overlaps with the 3 long terminal repeat (LTR) in the genome of HIV-1, HIV-2 or simian immunodeficiency virus (SIV). Nef is expressed early in the viral life cycle and is required for efficient viral replication and disease progression in the infected host. Therefore, the absence of Nef slows down or completely abolishes the progression towards acquired immunodeficiency syndrome (AIDS) $[1,2]$. Interestingly, a strong selective pressure has been demonstrated for a functional nef gene, since macaques infected with non-pathogenic, SIV nef-mutant show in vivo repair of the reading frame and subsequent progression to AIDS-like disease [3]. Moreover, isolated expression of Nef in transgenic mice induces a strong depletion of $\mathrm{CD}^{+}$cells, resembling an AIDS-like phenotype [4,5]. Additionally, cohorts of patients infected with HIV variants harboring Nef mutations and/or deletions in nef show a delayed onset of AIDS. While these studies clearly established Nef as a critical factor for AIDS pathogenesis, the underlying molecular mechanism remains to be fully elucidated.

Nef associates with host cell membranes through the $\mathrm{N}$-terminal myristoyl group and functions as an adaptor protein, promoting viral pathogenicity probably by interacting with several classes of host cell proteins, mainly protein kinases and components of the endocytic trafficking machinery. For instance, Nef reduces surface expression of the HIV entry receptor CD4 and coreceptors CCR5 and CXCR4 to prevent superinfection of already productively infected cells and possibly aiding virion release from these cells [5-9]. Nef also leads to reduced cell surface expression of $\mathrm{MHC}$ class I and $\mathrm{MHC}$ class II molecules to facilitate immune evasion of infected cells [6-8]. Finally, Nef interferes with the T cell receptor signal transduction machinery and enhances virion infectivity and viral replication $[9,10]$.

Mutational analysis revealed that individual actions of Nef have distinct structural correlates. The protein has a flexible, myristoylated N-terminal anchor domain of variable length followed by a loop section containing a proline-rich type II helix, a core domain and a C-terminal flexible loop containing an endocytic di-leucine based sorting motif that is required for most trafficking functions of Nef [11]. Specifically, downregulation of CD4 requires conserved amino acid residues located at the $\mathrm{N}$ terminal arm and the disordered C-terminal loop of Nef, whereas downregulation of MHC-I and CXCR4 depend on a cluster of acidic amino acid residues (EEEE, stretch of glutamic or aspartic acids) as well as the neighboring proline-rich motif that is part of a Src homology domain 3 (SH3) binding surface of Nef (PxxPxVPxRP, first four amino acids represented by $\operatorname{PxxP}, \mathrm{x}$ is an unspecified amino acid). A large number of cellular partners have been identified and for some of them the binding sites on Nef have been mapped $[11,12]$.

In this study, we analyzed a panel of HIV-1 and HIV-2 $n e f$ alleles derived from clinical HIV isolates and identified a nef allele that was naturally mutated in an amphipathic stretch of amino acids in the PxxP loop region, compromising both the acidic cluster and the prolinerich motif. Analysis of this and other Nef mutants revealed a crucial role of the three amino acid valine-glycine-phenylalanine (VGF) motif which links the acidic cluster to the proline-rich motif. Without affecting the stability of the protein, specific mutations of the VGF motif interfered with a wide variety of Nef functions which are known to depend on the integrity of the proline-rich motif, including MHC-I and CXCR4 downregulation, association of PAK2 and Hck kinases and interference with $\mathrm{T}$ cell receptor signaling as well as enhancement of viral infectivity and replication. For surface receptor trafficking and cytoskeletal functions each individual amino acid of the triplet is important; while for targeting of Lck kinase to the trans-golgi-network and viral infectivity, especially the phenylalanine of the triplet was essential. We propose that the highly conserved three amino acid VGF motif together with the adjacent acidic cluster and proline-rich motif form a previously unrecognized amphipathic surface on Nef that is essential for the majority of Nef functions.

\section{Results}

Nef alleles from different clades of HIV isolates modulate surface expression levels of human receptors

Nef downregulates the expression of cell surface markers, such as CD4, MHC-I and CXCR4. We initially set out to extend available data on the conservation of these functions [13-15] in a series of clinical HIV-1 (NIH AIDS Reference and Reagent Program) and HIV-2 isolates (Institute for Tropical Medicine, Antwerp, Belgium) that we used before to assess viral fitness hierarchy [16]. Nef alleles were sequenced (Additional file 1: Figure S1) and amplified from infected PBMCs and expressed by retroviral transduction in Jurkat CD4-CCR5 cells. Transduced cells were subsequently analysed by flow cytometry for surface receptor levels. Transduced cells express both Nef and an eGFP marker protein at correlating levels [17]. As a control, we included a virus encoding for eGFP only. In order to quantify the extent of receptor downregulation, the ratio between the mean fluorescence intensity (MFI) of transduced cells $\left(\mathrm{eGFP}^{+}\right)$was divided by the MFI of untransduced cells in the same culture. Expression of all nef alleles resulted in at least 5-fold decrease of CD4 surface expression, confirming the functionality of these Nef proteins. However, the effect of the isolated nef alleles on MHC-I and CXCR4 surface 
expression was more variable (Figure 1). The majority of the isolated HIV-1 group M, HIV-1 group O and HIV-2 Nef proteins downmodulated the expression of $\mathrm{MHC}-\mathrm{I}$ and CXCR4 resulting in a 2 - to 3 -fold reduction in surface expression. Interestingly, two HIV-1 subtype $\mathrm{C}$ alleles (VI1044 and VI794; Figure 1 and 1B) and one HIV-1 group $\mathrm{O}$ allele (O8) did not show any effect on $\mathrm{MHC}-\mathrm{I}$ downregulation (Figure 1C and 1D). All Nef proteins, except Nef O8 and HIV-2 VI171, downregulated the surface expression of CXCR4 (Figure 1C and 1D). In line with previous reports [15], nef alleles isolated from HIV-2 strains were much more efficient in downregulating CXCR4 than HIV-1 nef alleles (near 10-fold reduction of CXCR4 surface expression for HIV-2 compared to 2-3fold for HIV-1). Clearly, there was no correlation between the ability of various Nef proteins to downregulate CXCR4, MHC-I and CD4, confirming that the mechanisms of CD4 and MHC-I downregulations are at least in part genetically separable $[15,18,19]$ and also suggesting that downregulation of CXCR4 and MHC-I depend on the interaction of Nef with distinct cellular ligands and machineries.

\section{Mutational analysis of the natural Nef mutant HIV-1 08}

In our panel of primary nef alleles, we identified 4 nef alleles that were deficient for MHC-I and/or CXCR4 downregulation. A subtype C nef allele (VI1044) contained an insertion of a few amino-acids, which interrupted the structure of the $\mathrm{N}$-proximal $\alpha$-helical region, explaining the lack of MHC-I downregulation capacity of this allele (Additional file 1: Figure S1C) [20]. Nef alleles VI794 (subtype C) and VI171 (HIV-2) did not show any mutations in regions known to be important for Nef functions (Additional file 1: Figure S1C and Figure S1D). By contrast, Nef O8 showed mutations in an amphipathic stretch of amino acids in the Nef core domain encompassing the acidic cluster and the proline-rich motif (Figure 2A). More specifically, a point mutation changed the first proline of the polyproline stretch into an alanine. In addition to this point mutation in the PxxP motif, Nef O8 also showed a deletion of a three peptidic motif, i.e. valine-glycinephenylalanine (VGF), located right in between the acidic cluster and the polyproline stretch. Based on the sequences found in the Los Alamos HIV sequence database consulted in December 2011, the VGF was highly conserved in HIV-1 and SIVcpz (V: $99.20 \%$; G: 98.85 \%; F: $99.52 \%)$. [21,22]. HIV-2 nef alleles instead contain a highly conserved VGV motif (V: $93.06 \%$; G: $97.22 \%$; $: 79.17 \%$, [22]) indicating that these residues are important for optimal Nef function. Despite intensive research on the functions of conserved regions in Nef, the importance of the VGF/V region has not yet been appreciated or investigated thoroughly.
To evaluate the individual contribution of the observed mutations for the defective phenotype of the O8 nef allele, we generated a panel of mutants in which the different mutations were sequentially reverted to their consensus variant (Figure 2A) and re-evaluated their ability to downregulate surface molecules. Neither restoration of the AxxP sequence into PxxP (i.e. EDED_PxxP) nor introduction of the deleted amino acids (i.e. EDED_VGF_AxxP) restored the capacity to interfere with MHC-I and CXCR4 surface expression (Figure 2B and $2 \mathrm{C}$ ). Only simultaneous restoration of the PxxP and the VGF motif into the consensus variant (i.e. EDED_VGF_PxxP or EEEE_VGF_PxxP) rescued CXCR4 and $\mathrm{MHC}-\mathrm{I}$ downregulation by $\mathrm{O} 8 \mathrm{Nef}$. Interestingly, when all three amino acids of the VGF motif in Nef O8 were converted into alanine (i.e. EDED_AAA_PxxP), in the presence of an intact polyproline stretch, the capacity to affect MHC-I and CXCR4 surface expression was again lost (Figure $2 \mathrm{~B}$ and $2 \mathrm{C}$ ), indicating that specific amino acids in both motifs are important.

\section{An amphipathic surface in Nef is important for downregulation of CXCR4 and MHC}

Depiction of the VGF motif within the structure of Nef showed that this motif is located within a loop region of Nef directly preceding the PxxP motif (Figure 3A). The motif is at the surface of Nef and highly accessible for protein-protein interactions [23]. To prove the functional conservation of the VGF motif, we initially evaluated the importance of this domain for MHC-I and CXCR4 downregulation in nef alleles derived from HIV1 group $M$, two canonical group (M) subtype B strains (i.e. NL4.3 and NA-7) and two non-canonical isolates: subtype B (i.e. B2) and subtype C (i.e. VI1422). Mutations were designed to generate triple mutations of VGF into alanines (VGF $\rightarrow$ AAA).

To exclude the possibility that the introduced mutation could affect the stability of the Nef protein, we transduced Jurkat T cells to express wild type (WT) Nef proteins and their mutant counterparts and evaluated their expression by Western blotting (Figure 3B). Equal amounts of Nef protein were present in sorted cell lysates, indicating that the VGF to AAA mutation did not affect the stability of the protein. Next, we evaluated the influence of these mutant Nef proteins on surface receptor expression. Analysis of the surface expression of CD4, CXCR4 and MHC-I by flow cytometry did not reveal a deleterious effect of $\mathrm{VGF} \rightarrow \mathrm{AAA}$ mutation on CD4 downregulation of all nef alleles analyzed, indicating that the expressed Nef mutants retained this function (Figure 3C), as did O8 Nef. However, these mutants were completely defective for MHC-I and CXCR4 downregulation (Figure $3 \mathrm{D}$ and $3 \mathrm{E}$ ). This finding indicates that the VGF motif is not only important for downregulation of 


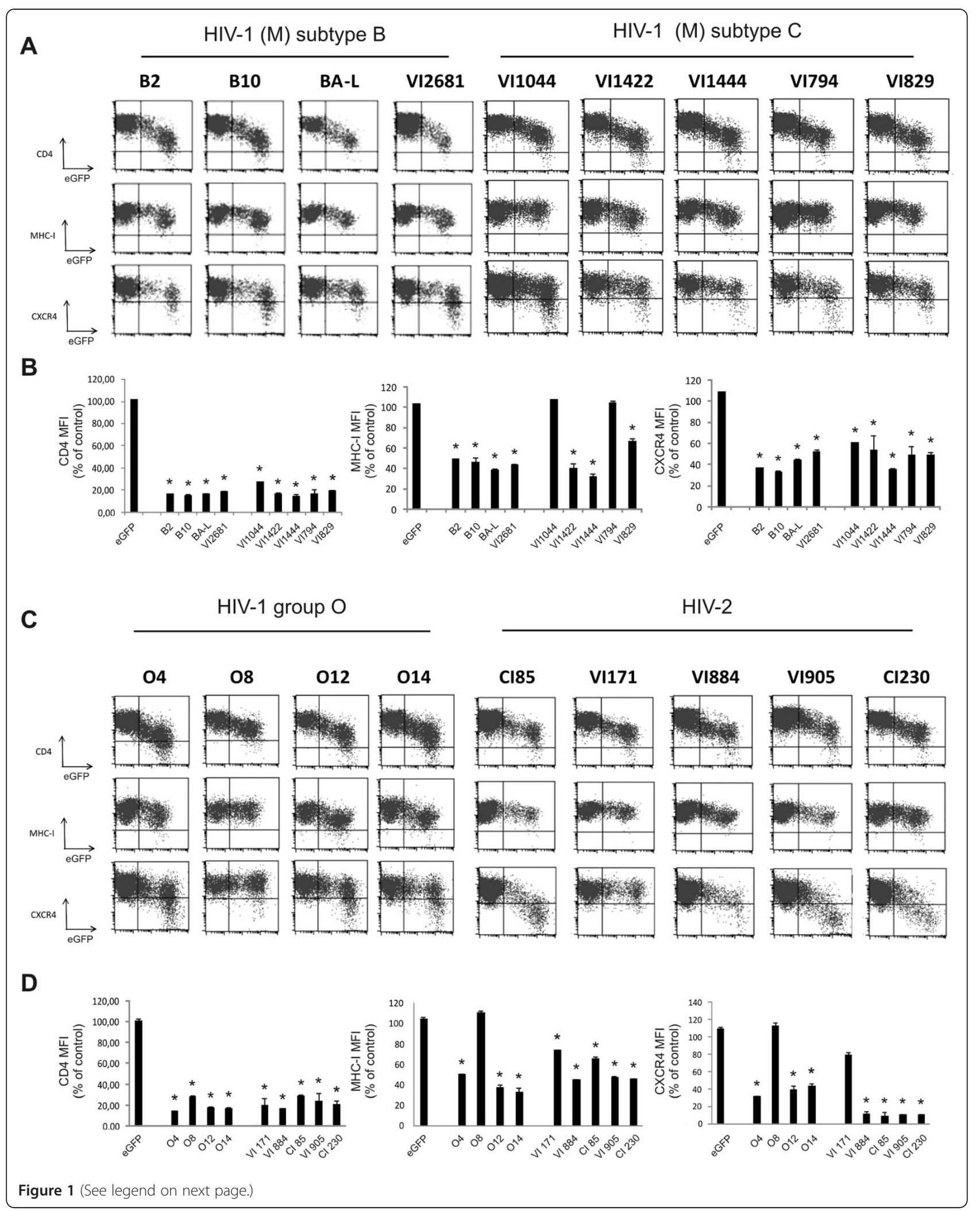


(See figure on previous page.)

Figure 1 Effect of primary HIV-1 and HIV-2 Nef proteins on surface receptor expression. Jurkat CD4-CCR5 cells were transduced with retroviral vectors expressing the indicated nef alleles and eGFP (Nef-IRES-eGFP) and were analyzed by flow cytometry. (A and C) Bivariate dot plots show expression of CD4, MHC-I, CXCR4 as a function of eGFP (Nef) expression. Dot plots shown are representative of results obtained in at least 3 independent experiments. (B and D) To determine relative CD4, MHC-I and CXCR4 surface levels, the MFI of transduced cells (eGFP ${ }^{+}$) was divided by the MFI of untransduced cells (eGFP') in the same culture. Data represent the mean relative expression and standard deviations from at least 3 independent experiments. eGFP represents the value for control transduced cells expressing only the marker gene but no Nef. An asterisk $\left(^{*}\right)$ over a bar indicates a statistically significant difference between the experimental construct compared with the eGFP control (P $\left.<0.01\right)$.

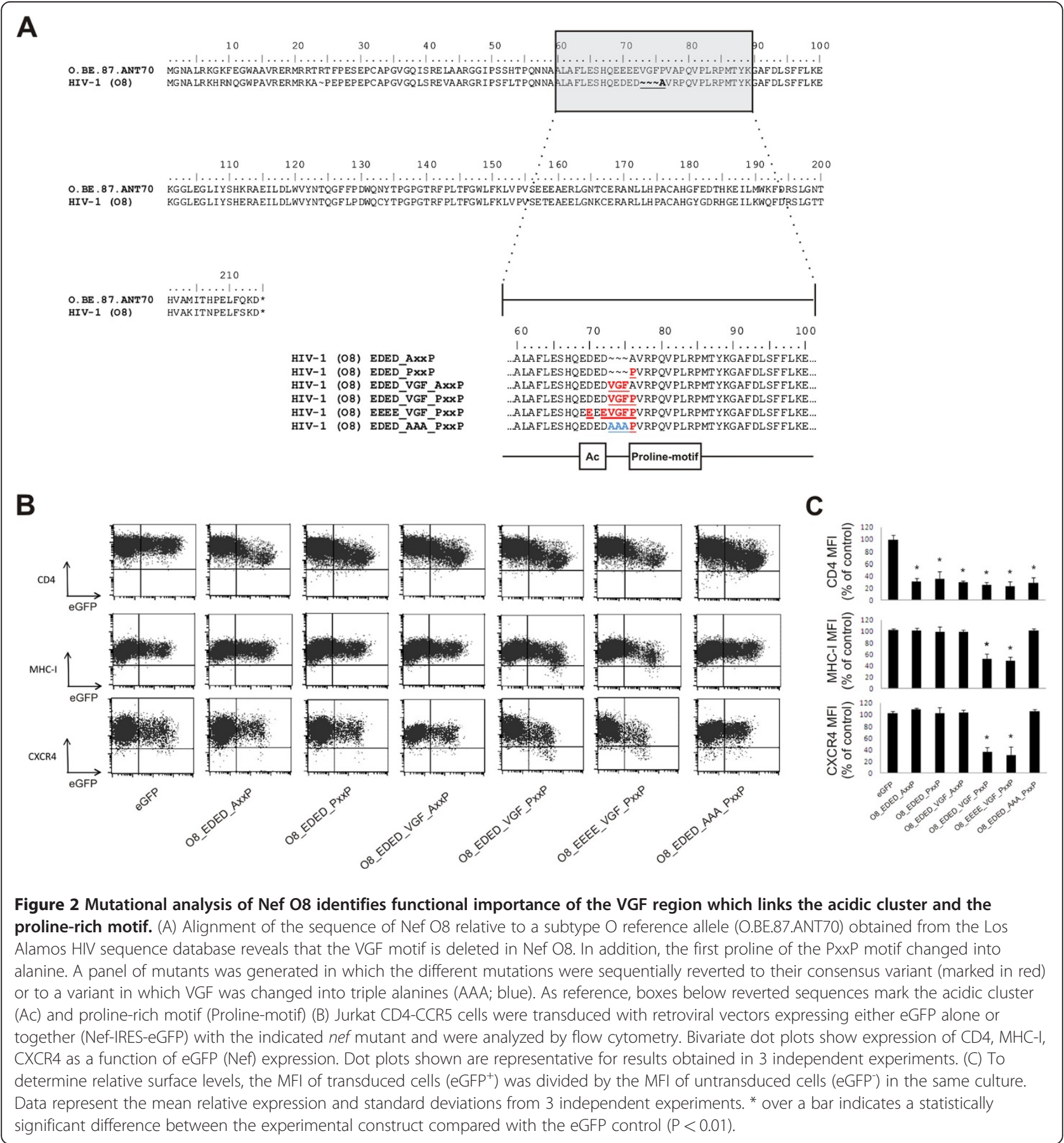



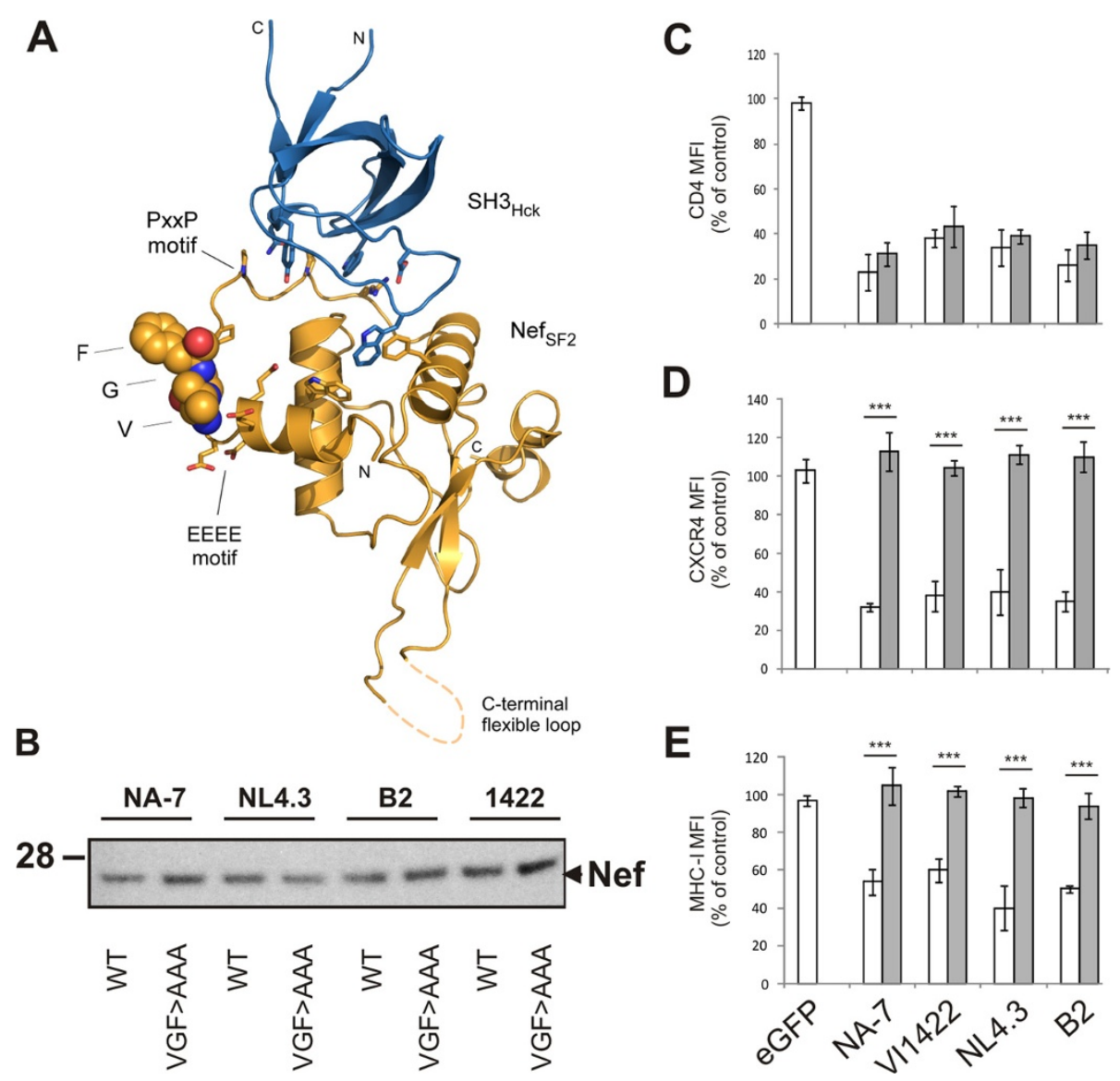

Figure 3 The VGF linker region is critical for downregulation of CXCR4 and MHC-I by HIV-1 Nef. To evaluate the functional importance, $\mathrm{VGF} \rightarrow$ AAA mutants of several Nef proteins were expressed and evaluated for their effect on cell surface receptor expression. (A) Display of the VGF motif in the structure of Nef $\mathrm{SF}_{2}$ (yellow ribbon) bound to an Hck-SH3 domain (blue ribbon). VGF residues, which are deleted in Nef allele O8, are indicated as space-filling model. Residues of the preceding acidic cluster and the following PxxP motif are shown as stick representation. The structure is based on PBD accession number 3REA [23]. (B) WT or VGF $\rightarrow$ AAA Nef-IRES-eGFP transduced Jurkat CD4-CCR5 cells were sorted, lysed and analyzed for Nef expression. The figure shows a Western blot of whole cell lysates stained with sheep-anti-Nef antiserum. The marker line indicates position of a 28 kDa standard. Loading control staining (actin) was similar over all samples (not shown). (C-E) Relative CD4, MHC-I, CXCR4 surface levels measured by flow cytometry on Jurkat CD4-CCR5 cells transduced with retroviral vectors expressing either eGFP alone or together with the indicated nef alleles and mutants. To determine relative surface levels, the MFI of transduced cells (eGFP+) was divided by the $\mathrm{MFI}$ of untransduced cells (eGFP') in the same culture. Data represent the mean relative expression and standard deviations obtained from 3 independent experiments, wild-type allele (open columns) next to the corresponding VGF $\rightarrow$ AAA mutants (grey shaded columns). ${ }^{* *}$ over a bar indicates a statistically significant difference between the VGF $\rightarrow$ AAA mutant compared to the wild-type allele (P $<0.001)$.

these surface molecules in Nef O8, but also in HIV-1 group M Nef proteins.

Each amino acid residue of the VGF motif is highly conserved, suggesting a sequence specific requirement. However, the triple mutation of the VGF stretch might be structurally drastic and alter the functionality of adjacent motifs. To exclude this and to confirm the sequence specific requirement of the VGF motif, a panel of single mutants of the VGF motif, in which the different amino acids are changed into alanine (AGF, VAF and VGA), was generated and evaluated for their effect on Nef function. Similarly to the VGF $\rightarrow$ AAA mutant, single mutants failed to down regulate MHC-I and CXCR4, while maintaining the capacity to downregulate CD4 (Figure 4). These results indicate that the VGF stretch as such is required for downregulation of MHC-I and CXCR4.

Integrity of the VGF motif is essential for effects of Nef on actin dynamics and Lck localization

Due to the spatial proximity of the VGF motif to the polyproline stretch containing the PxxP-motif, we wondered whether both motifs support distinct functions of Nef or are integrated into one larger protein binding surface. To address this point we analyzed Nef functions in addition to MHC-I and CXCR4 downregulation known to depend on the PxxP motif and focused first on the 


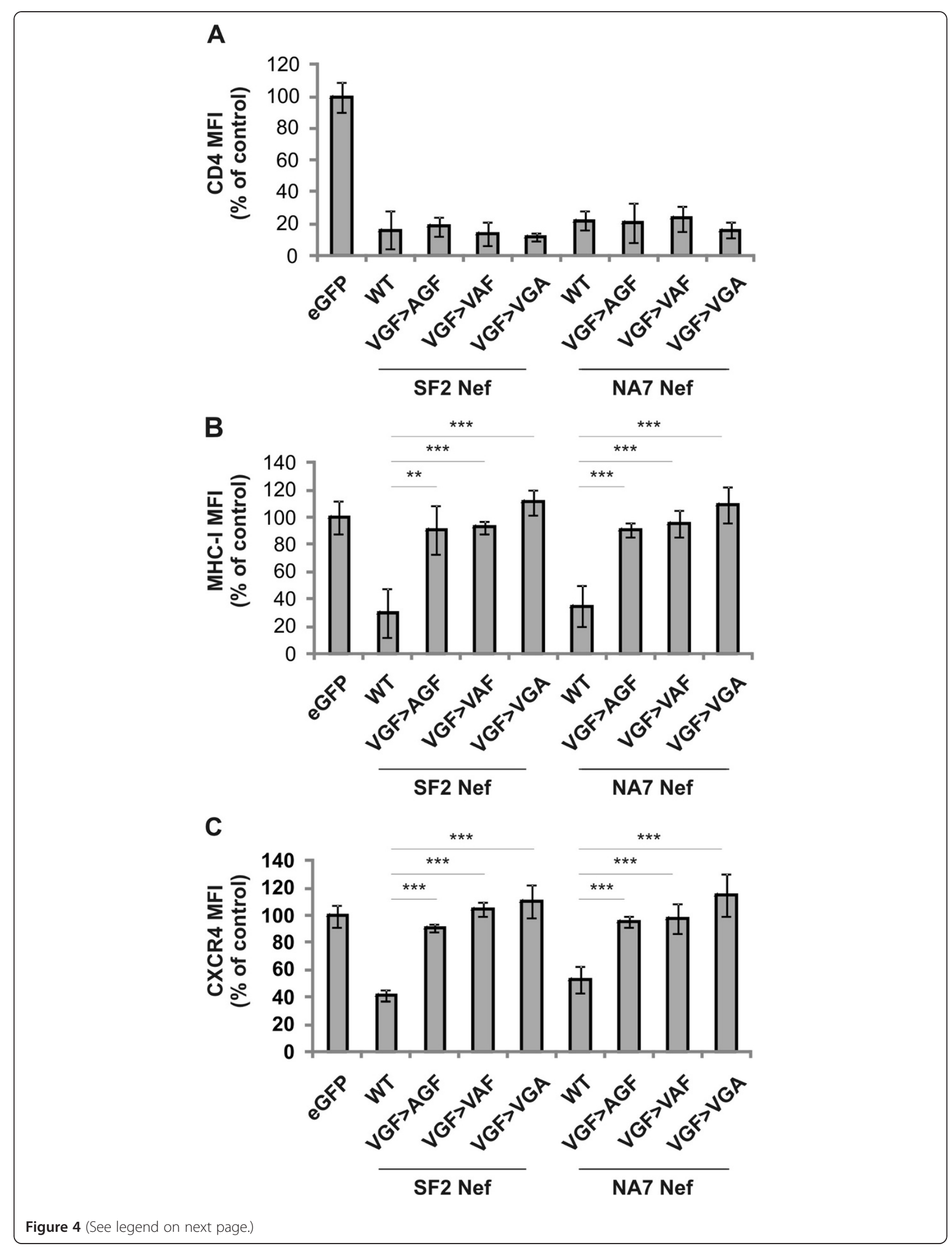


(See figure on previous page.)

Figure 4 Sequence specific requirement of V-G-F for Nef function. Panels A to C show relative surface levels of CD4, MHC-I, CXCR4 respectively, measured by flow cytometry on Jurkat CD4-CCR5 cells transduced with retroviral vectors expressing either eGFP alone or together with the indicated nef alleles and mutants. To determine relative surface levels, the MFI of transduced cells (eGFP ${ }^{+}$) was divided by the MFI of untransduced cells (eGFP) in the same culture. Data represent the mean relative expression and standard deviations obtained from 3 independent experiments, ${ }^{* *}$ over a bar indicates $P<0.01$ and ${ }^{* * *}$ indicates $P<0.001$.

association of Nef with the cellular p21-associated kinase 2 (PAK2) [24]. Nef-PAK2 association occurs in a highly labile multiprotein complex of about $1 \mathrm{MDa}$ and causes the phosphorylation and thereby inactivation of the actin severing factor cofilin [25,26]. While the instability of the Nef-PAK2 complex limits the reliability of biochemical quantification of this association between different nef alleles [24,26,27], the functional consequences including PAK2-dependent deregulation of cofilin and inhibition of actin dynamics can readily be quantified and are conserved among most Nef isolates [26,28]. We therefore analyzed the ability of Nef VGF $\rightarrow$ AAA mutants from SF2 and NA7 Nef to induce hyper-phosphorylation of cofilin downstream of PAK2, using a previously established single cell-based immunofluorescence quantification assay $[20,21]$. As expected, cofilin was hyper-phosphorylated in Jurkat T cells expressing WT SF2 and NA-7 Nef, but not in the presence of their AxxA mutants. Similarly, $\mathrm{VGF} \rightarrow \mathrm{AAA}$ mutated Nef failed to induce cofilin phosphorylation (Figure 5A and $5 \mathrm{~B}$ ), suggesting that the VGF motif is essential for Nef-PAK2 association. To address this point directly, we performed an in vitro kinase assay to analyze the association of Nef with PAK2 activity. GFP-tagged SF2 Nef was used for this analysis due to more robust kinase signal detected with this Nef allele. Nef.GFP was immunoprecipitated from transiently transfected Jurkat $\mathrm{T}$ cells and subsequently subjected to an in vitro kinase assay, in which Nef-PAK2 association is detected by the presence of autophosphorylated PAK2 (62 kDa; p-PAK2) as well as of a yet unindentified $72 \mathrm{kDa}$ substrate (p72). Expectedly [26,29], WT SF2 Nef but not its AxxA mutant efficiently associated with autophosphorylating PAK2; however, SF2 AxxA Nef was also expressed to lower levels than the WT in the experiment shown. In line with the results obtained from the cofilinphosphorylation analyses, the VGF Nef mutant failed to associate with PAK2 activity despite robust expression and efficient immune isolation (Figure 5C). Since the deregulation of cofilin by Nef results in impaired host cell actin remodeling following external stimulation, we next analyzed the role of the VGF motif for the Nef-dependent inhibition of actin remodeling following TCR-signaling $[28,30,31]$. T cells transiently expressing GFP-tagged versions of WT, AxxA or VGF $\rightarrow$ AAA mutants from SF2 or NA7 Nef were plated onto anti-CD3 coated cover glasses and analyzed for the formation of circumferential F-actin rich rings by immuno fluorescence analysis. Expectedly,
WT Nef expressing cells interfered with actin remodeling and prevented the formation of F-actin rich circumferential rings: as shown in Figure 4D, no actin ring is visible around green cells expressing WT Nef. In contrast, AxxA and $\mathrm{VGF} \rightarrow \mathrm{AAA}$ Nef variants were not able to interfere with actin polymerization (Figure $5 \mathrm{D}$ and $5 \mathrm{E}$ ), so a clear circumferential staining is visible, despite Nef mutant protein expression.

Similar to the VGF $\rightarrow$ AAA mutants, single mutants of the VGF motif (AGF, VAF and VGA) were significantly reduced in their effect on cofilin phosphorylation (Figure $5 \mathrm{~A}$ and $5 \mathrm{~B}$ ) and actin polymerization (Figure 5D and $5 \mathrm{E})$. It should, however, be noted that these single mutants had a slight but significant residual effect as compared to the triple mutant, indicating that some residual activity remains for the alleles, especially for the $\mathrm{VGF} \rightarrow \mathrm{AGF}$ and $\mathrm{VGF} \rightarrow \mathrm{VAF}$ mutants. Nevertheless, these results demonstrate that the VGF motif in different nef alleles is critical for Nef-PAK2 association and thus consequential for Nef-mediated cofilin deregulation and disruption of host cell actin remodelling.

The above results demonstrated for different nef alleles a requirement for both the PxxP and the VGF motif in Nef-PAK2 association and cell surface receptor down modulation. All of these activities, however, also depend on additional protein interaction motifs in Nef such as an intact protein interaction surface around aa191/195 or the acidic cluster [32] and thus did not allow us to conclude unambiguously whether the VGF and PxxP motifs exert independent functions or synergize in a common molecular interaction. We, therefore, assessed next the role of the VGF motif in Nef-mediated targeting of the TCR proximal kinase Lck to the trans-golgi network (TGN), a function of Nef for which so far no protein interaction surface in Nef other than the the PxxP motif has been implicated [30,33,34]. Jurkat T cells transiently expressing GFP-tagged Nef proteins were analyzed for TGN accumulation of Lck by immunofluorescence analysis. While Lck appeared mostly at the plasma membrane with a minor intracellular fraction in GFP- and Nef AxxA-expressing cells, WT Nef expression resulted in a pronounced intracellular accumulation indicative of TGN targeting of the kinase (Figure 6A) as described previously [33]. $\mathrm{VGF} \rightarrow \mathrm{AAA}$ Nef mutants displayed intermediate activity in this assay and failed to affect the subcellular localization of Lck in most cells; however, some cells 


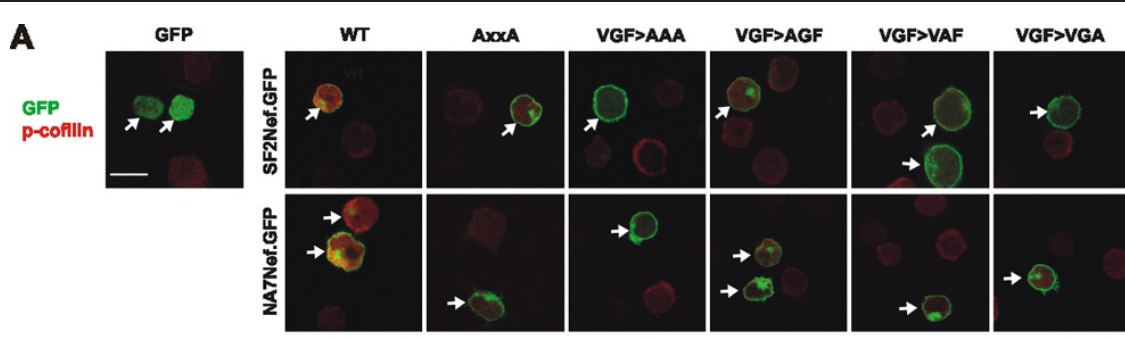

B

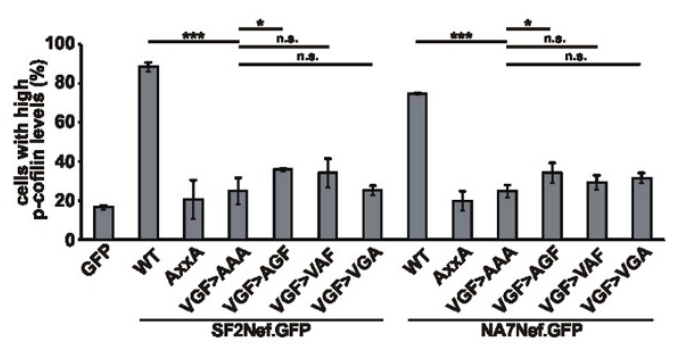

C

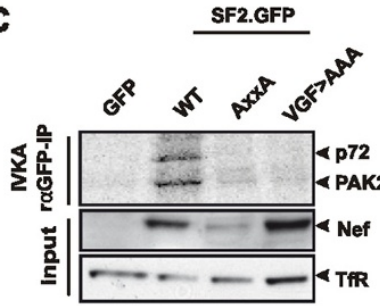

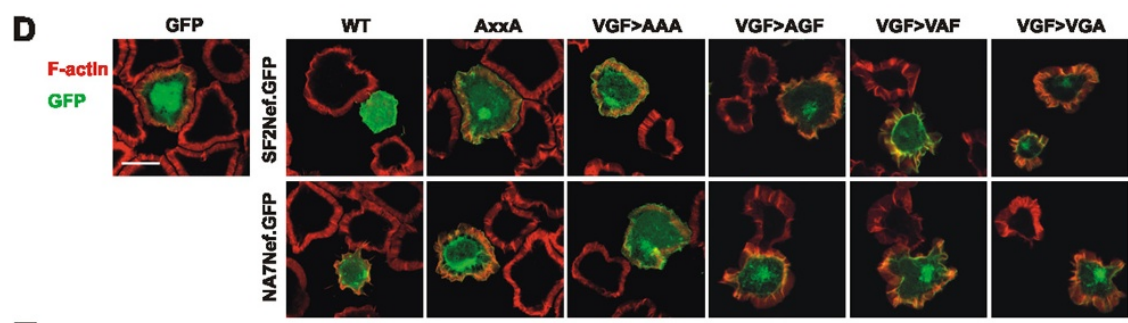

$\mathbf{E}$

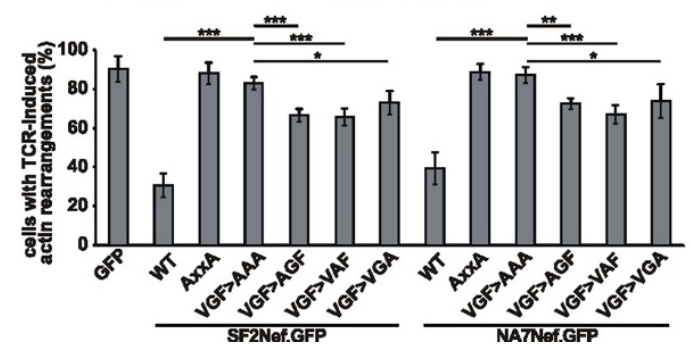

Figure 5 The VGF region is important for Nef-PAK2 association, cofilin hyper- phopsorylation and inhibition of F-actin rich circumferential ring formation by Nef. (A) Jurkat TAg cells were transfected arrows with WT, AxxA, VGF $\rightarrow A A A, V G F \rightarrow A G F, V G F \rightarrow V A F$ or $V G F \rightarrow V G A$ mutant Nef.GFP fusion proteins, plated onto poly-L-lysine (PLL)-coated cover glasses, fixed and stained for phospho-cofilin ( $p$-cofilin). Images show intense staining for p-cofilin (red-orange) only in cells expressing Nef (green) of wild-type, but not of mutated sequence. Scale bar $=10 \mu \mathrm{m}$ (B) Frequency of the cells from cultures as shown in panel A that were scored high in p-cofilin. Values are the means of 3 independent experiments, and error bars represent SD from the mean; $\geq 100$ cells were analyzed per transfection; * indicates $P<0.01$, ** indicates $P<0.001$ and ${ }^{* *}$ indicates $P<0.0001$. (C) Lysates of Jurkat TAg cells expressing eGFP or WT, VGF $\rightarrow A A A$ or AxxA SF2 Nef.GFP fusion proteins. Lower panels (input) show Western blot for Nef and loading control (transferrin receptor). These lysates were immunoprecipitated for Nef.GFP (raGFP-IP), and subsequently used for in vitro kinase assay (IVKA). Nef-PAK2 association is detected in the IVKA only with WT Nef, by the presence of autophosphorylated PAK2 (62 kDa; p-PAK2) as well as of a yet unindentified $72 \mathrm{kDa}$ substrate (p72). (D) Jurkat TAg cells were transfected with WT, AxxA, VGF $\rightarrow$ AAA, VGF $\rightarrow$ AGF, VGF $\rightarrow$ VAF or VGF $\rightarrow$ VGA mutant Nef.GFP fusion proteins, plated onto anti-CD3-coated cover glasses, fixed and stained with phalloidin to reveal F-actin (red). Scale bar $=10 \mu \mathrm{m}$ (E) Frequency of the cells from cultures as shown in panel A that to form F-actin-rich circumferential rings. Values are the means of 3 independent experiments, and error bars represent SD from the mean; $\geq 100$ cells were analyzed per transfection, ${ }^{*}$ indicate $\mathrm{P}<0.01,{ }^{* *}$ indicates $\mathrm{P}<0.001$ and ${ }^{* * *}$ indicates $\mathrm{P}<0.0001$.

still showed slight TGN recruitment of Lck (Figures $6 \mathrm{~A}$ and $6 \mathrm{~B})$. In contrast, single substitutions of the valine $(\mathrm{VGF} \rightarrow \mathrm{AGF})$ and the glycine $(\mathrm{VGF} \rightarrow \mathrm{VAF})$ respectively did not or did marginally affect Lck accumulation. However, mutation of the phenylalanine,
$(\mathrm{VGF} \rightarrow \mathrm{VGA})$ was sufficient to abrogate this Nef function similar to the VGF $\rightarrow$ AAA mutant, indicating that Lck accumulation by Nef depends at least in part on the VGF motif and that the phenylalanine which directly precedes the proline-rich stretch is crucial for this. 

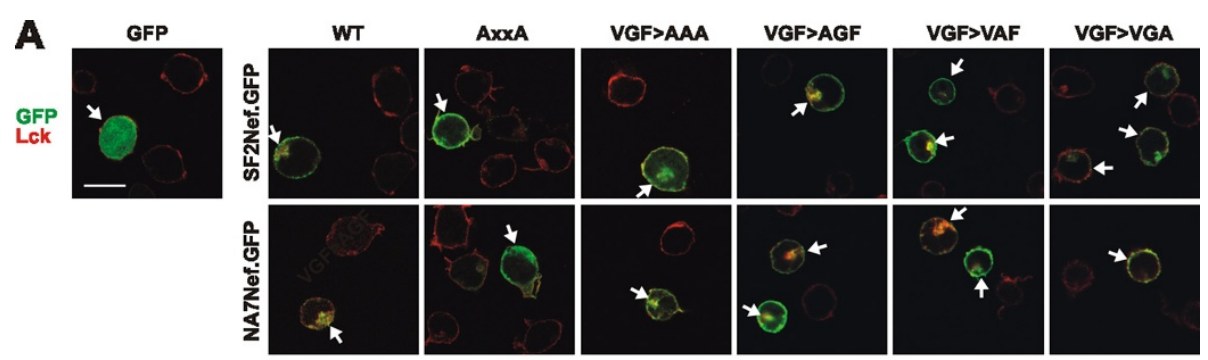

B

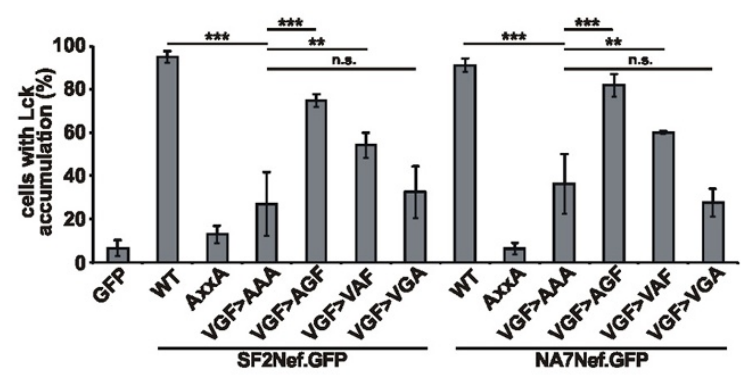

C


Figure 6 The VGF region is important for the interaction of Nef with Lck and Hck. (A) Jurkat TAg cells were transfected arrows with WT, $A x x A, V G F \rightarrow A A A, V G F \rightarrow A G F, V G F \rightarrow V A F$ or VGF $\rightarrow$ VGA mutant Nef.GFP fusion proteins, plated onto poly-L-lysine (PLL)-coated cover glasses, fixed and stained for Lck (red): wild-type but not AxxA Nef induces Lck accumulation (arrow heads), Images shown are representative for all cells analyzed. Scale bar $=10 \mu \mathrm{m}$ (B) Frequency of the cells from cultures as shown in panel A that show Lck accumulation. Values are the means of 3 independent experiments, and error bars represent SD from the mean; $\geq 100$ cells were analyzed per transfection, ${ }^{* *}$ indicates $P<0.001$ and *** indicates $P<0.0001$. (C) Western blot of lysates of 293 T cells co-expressing WT, VGF $\rightarrow A A A, V G F \rightarrow A G F, V G F \rightarrow V A F, V G F \rightarrow V G A$ or $A x x A$ SF2 or NA-7 Nef.GFP fusion proteins (all similar fraction of cells positive for GFP) or GFP only together with Hck. Lower panels (input) show staining for Nef (arrow) and Hck; upper panel shows anti-GFP immunoprecipitation (IP) stained for Hck. Interaction of Nef with Hck was detected as the presence of Hck isoforms in the immunoprecipitate.

To address whether the VGF motif is directly involved in protein interactions of the adjacent PxxP motif, we probed its impact on the direct interaction of Nef with its best characterized and highest affinity PxxP ligand, the Src-family kinase Hck [35]. Nef and Hck were coexpressed in 293 T cells and Nef.GFP was immunoprecipitated. Subsequently, Co-precipitating Hck was detected by Western Blot. While different isoforms of Hck co-precipitated efficiently with WT (NA-7 or SF2) Nef, the AxxA and VGF $\rightarrow$ AAA Nef mutants were completely defective in Hck binding. Single amino acid substitution mutants did not bind the low molecular weight isoform as WT preferentially did, although some residual binding was sometimes observed with the high molecular weight isoform (Figure 6C). Together, we concluded that the integrity of the VGF motif is essential for most, if not all, functions attributed to the PxxP motif and that both motifs may be part of a common interaction surface.

The VGF motif is essential for the effect of Nef on viral infectivity and replication

Nef has been shown to positively affect the replication of HIV in vivo [2] as well as in cultures of primary cells [36-38] and ex vivo human lymphoid tissue [39]. Multiple studies have shown that Nef positively affects viral replication by enhancing viral release and infectivity [10]. Since these functions critically rely on the PxxP motif, we addressed the replication and infectivity of $\mathrm{VGF} \rightarrow \mathrm{AAA}$ 
mutant HIV. We constructed a panel of replication competent $\mathrm{HIV}_{\mathrm{NL4.3}}$-based reporter viruses expressing NL4.3 WT, AxxA and VGF $\rightarrow$ AAA Nef. These viruses express Nef together with mouse-derived heat stable antigen (HSA) from a single bicistronic mRNA (HSA-IRES-Nef) [40]. It was shown before that key activities of Nef such as enhancement of virion infectivity and increase of viral replication remain conserved in such chimeric viruses and that marker gene expression correlates with Nef expression in productively infected cells $[41,42]$. As control, we also constructed a $\mathrm{Nef}_{\text {stop }} \mathrm{HIV}$ reporter virus, which does not express Nef due to the insertion of multiple premature stop codons [43].

We evaluated whether the $\mathrm{VGF} \rightarrow \mathrm{AAA}$ mutation affected the infectivity of HIV particles in HeLa P4R5 $\beta$ galactosidase indicator cells. Cells were infected with virus preparations corresponding to equal amounts of p24 and HIV-infection was quantified on the basis of $\beta$ galactosidase activity, $48 \mathrm{~h}$ post infection (Figure 7A). As expected, the $\mathrm{Nef}_{\text {stop }} \mathrm{HIV}$, deficient in Nef expression

\section{a}

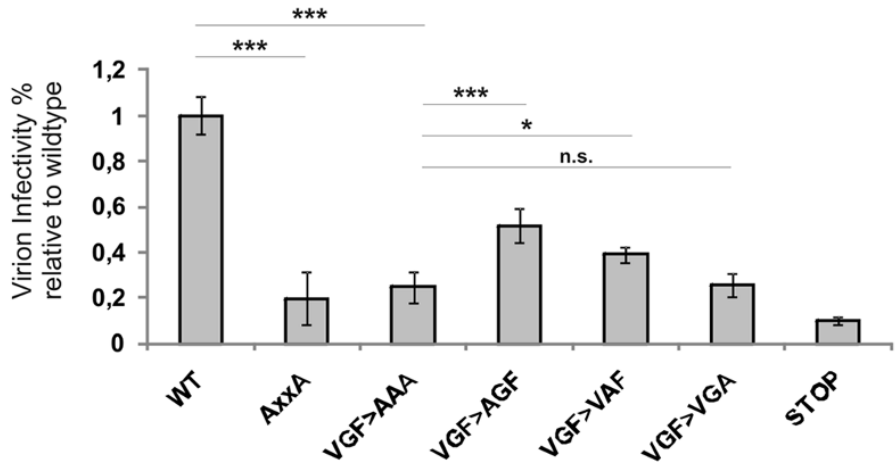

b

Donor 1
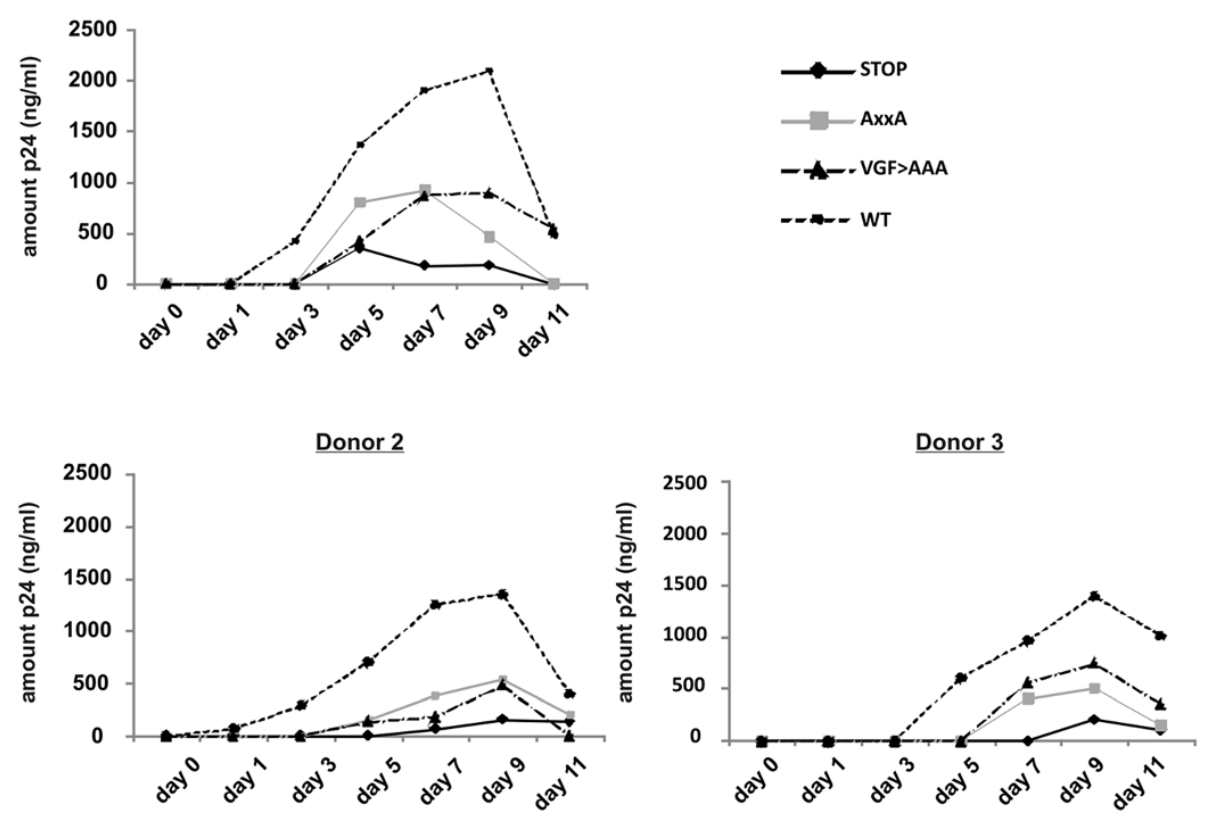

Figure 7 Integrity of the VGF region is required for enhancement of virus infectivity and replication by Neflntegrity of the VGF region is required for enhancement of virus infectivity and replication by Nef. Replication competent HIV-1 NL4.3 HSA-IRES-Nef reporter viruses (either Nef WT, AxxA, VGF $\rightarrow$ AAA, VGF $\rightarrow$ AGF, VGF $\rightarrow$ VAF, VGF $\rightarrow$ VGA, or Stop) were produced in 293 T cells and quantified by p24 measurement (A) Enhancement of virion infectivity by different Nef mutants in HeLa P4R5 LTR- $\beta$-galactosidase indicator cells. Percentage $\beta$-gal levels were calculated relative to wild-type HIV-1 NL4-3 HSA-IRES Nef. Values are the means of 3 independent experiments, and error bars represent SD from the mean; * indicates $P<0.05$, ${ }^{* *}$ indicates $P<0.01$ and ${ }^{* *}$ indicates $P<0.001$. (B) Replication kinetics in human primary CD4+ T cells. Freshly isolated peripheral blood CD4+ T cells were stimulated for $48 \mathrm{~h}$ with IL-2/PHA before infection. After 1, 3, 5, 7, 9 and 11 days of culture, HIV replication was monitored by determining the amount of p24 antigen in the supernatants. Plots show the replication kinetics of the HIV reporter virus in CD4+ T cells derived from 3 different donors. 
was almost 8-fold less infectious than HIV-1 WT virions. Both the VGF $\rightarrow$ AAA and AxxA Nef mutated HIV were severely less infective. Results shown were obtained with the NL4-3 nef allele and similar reduction in infectivity was seen using SF2 and NA-7 nef alleles (data not shown). Similary to the VGF $\rightarrow$ AAA mutant of NL4.3 Nef, single mutants were significantly less infective relative to the wt virus. Of note, single substitution mutants of the valine $(\mathrm{VGF} \rightarrow \mathrm{AGF})$ and the glycine $(\mathrm{VGF} \rightarrow \mathrm{VAF})$, but not of the phenylalanine (VGF $\rightarrow \mathrm{VGA}$ ), were slightly more infective than the triple mutation, resulting in an intermediate phenotype.

As a final functional correlate, we evaluated if the integrity of the VGF motif is essential for Nef enhanced HIV replication in peripheral blood lymphocytes (PBL). CD4+ T cells were isolated and stimulated with IL-2/ PHA, prior to infection with isogenic viruses. Cells were infected with 293 T-derived viral supernatants corresponding to the same amount of p24. After 1, 3, 5, 7, 9 and 11 days of culture, HIV replication was monitored by determining the amount of p24 antigen in the supernatants (Figure 7B). WT NL4.3 HIV-1 replicated much more efficiently in IL-2 stimulated PBL's relative to the nef stop variant. As expected the mutants of the PxxP motif were less efficient in enhancing viral replication [44,45]. Similarly, the VGF mutated Nef HIV replicated less efficiently compared to WT HIV, although the effect was attenuated in some donors when compared to the PxxP Nef mutated HIV. All donors tested showed a significantly reduced replication rate of $\mathrm{HIV} \mathrm{VGF} \rightarrow \mathrm{AAA}$ Nef mutated virus compared to WT Nef HIV. After day 9 of culture, viability in infected cultures decreased significantly, explaining the general drop in p24 levels at day 11. As a control, we also evaluated the amount of HSA expressing cells at several time points by flow cytometry as an alternative measure for HIV expression, with similar results (data not shown).

\section{Discussion}

Although not essential for viral replication, HIV-1 Nef is important for disease progression and, therefore, is considered a pathogenic factor in primate lentiviridae [10]. For its function, Nef depends on specific surfaces to interact with host cell proteins. Therefore, a strong positive selection pressure exists to keep the property of these surfaces conserved [46]. It is key to identify conserved sites in Nef with functional importance in protein-protein interactions, since they might serve as potential target sites for pharmacological intervention.

In the present study, we identified, by functional analysis of a panel of clinical HIV-1 and HIV-2 nef alleles, a previously unrecognized conserved region. A HIV-1 group $\mathrm{O}$ nef allele, was mutated in an amphipathic stretch of amino acids located in the core domain encompassing the acidic cluster, a VGF region and the PxxP motif, resulting in the loss of downregulation of MHC-I and CXCR4, but not of CD4. By selective mutation of this $\mathrm{O} 8$ nef allele back to the consensus sequence, we show that both an intact PxxP motif in conjunction with an intact VGF region are needed to restore the defect in receptor trafficking. We could extend this observation by showing that the VGF region is essential for the association of Nef with active PAK2 and consequently hyperphosphorylation of cofilin resulting in the inhibition of actin remodeling following TCR triggering. In addition, Nef-induced Lck accumulation in the TGN also requires integrity of the VGF region. The importance of the proline-rich region for this Nef function has been studied extensively $[10,47]$. However, despite its high conservation in HIV-1 and SIVcpz nef alleles, the function of the VGF region remained poorly investigated.

Modulation of cell surface molecules such as MHC-I and chemokine receptors or the TNF receptor-associated factor TRAF2 were shown to depend on the conservation of the PxxP motif as well as the acidic cluster (EEEE) $[19,48,49]$. How the acidic cluster contributes to downregulation of MHC-I is controversial. Several studies have observed that the four contiguous glutamate residues interact with PACS-1 and PACS-2 to initiate assembly of a multiprotein complex which targets MHC-I to the trans-Golgi network for subsequent degradation [50,51]. However, in an alternative model for MHC-I downregulation, in which Nef interacts with both MHCI and the $\mu$ subunit of the AP- 1 endosomal coat complex, the EEEE motif plays only a stabilizing role $[52,53]$. The PxxP motif on the other hand is essential for the interaction of Nef with Src kinases (like Hck); misrouting of Lck as well as association of Nef with the cellular kinase PAK2, results in elevated cellular levels of inactivated phosphorylated cofilin, a deregulation that is instrumental for the inhibition of TCR- or chemokineinduced F-actin remodeling [26,28-31,34].

Triple mutants of the VGF motif (VGF $\rightarrow$ AAA) lost both 'trafficking' (MHC-I, CXCR4) as well as 'signaling' (PAK2 association, cofilin hyper-phosphorylation and inhibition of actin ring formation) functions, suggesting that the VGF region is functionally linked with the PxxP motif. Moreover, Baugh et al. showed that similar to the PxxP motif, MHC-I downregulation, interaction with PAK2, and to a lesser degree enhancement of virion infectivity are dependent on the acidic cluster [32]. Our observation that mutation of VGF into triple alanines similarly abrogates these multiple effects of Nef is a hint that the acidic cluster-VGF-PxxP triplet forms a functional unit important for protein-protein interactions with host cell factors.

In the dysfunctional Nef O8 allele, the VGF motif is in fact deleted, but not replaced to varying amino acids by gene tropism. The effect of the deletion mutation could 
therefore be indirect, meaning that only the correct spacing between the acidic cluster EEEE motif and the PxxP motif is required for Nef functionality, i.e. the correct positioning of the EEEE motif and potentially the preceding CAWL protease recognition motif with respect to the Nef core domain. Alternatively, the effect of Nef dysfunction could be direct through binding of these amino acids to the primary receptors itself or to any stimulatory co-factor required for receptor downregulation. As mutation of the VGF motif to AAA in O8 Nef did not restore the Nef trafficking functions, the effect of mutation is most likely direct. Our finding that individual mutation of the three amino acids (AGF, VAF, VGA) is sufficient to interfere with the effect of Nef on receptor trafficking and TCR signaling further supports this hypothesis and shows that the function of V-G-F is sequence specific. The integrity of the phenylalanine appears to be specifically essential for functions related to the proline-rich motif. In contrast to single mutants of the valine and the glycine (VGF $\rightarrow$ AGF and $\mathrm{VGF} \rightarrow \mathrm{VAF}$ ), single mutants of this amino-acid (VGF $\rightarrow \mathrm{VGA}$ ) did not accumulate Lck. Of note, several $\mathrm{SH} 3$ binding regions of mammalian proteins contain a $\mathrm{N}$-terminal phenylalanine, which is required for efficient ligand binding [54]. These observations suggest that the effect of Nef on TCR induced actin remodeling and on Lck targeting to the TGN are mechanistically different, as was shown before [30].

Shelton et al. showed recently that the VGFPV region, in their study identified as the secretion modification region, forms a specific binding surface for mortalin which subsequently promotes cellular secretion of extracellular Nef [55]. The phenotypes which we analysed in this study are all cell intrinsic and are unlikely to be explained by differences in levels of extracellular Nef. The results of Shelton et al. and ours together show that the VGF region is not just a mere spacer between the acidic cluster and the proline rich region. Instead, it acts as an interaction surface, which together with its neighboring motifs forms a functional unit.

Replication of HIV-1 in primary $\mathrm{T}$ lymphocytes is tightly coupled to their activation state. While HIV-1 undergoes early replication events in quiescent $\mathrm{CD} 4+\mathrm{T}$ cells, subsequent steps in the viral life cycle require $\mathrm{T}$ cell activation. One of the prime roles of Nef in vivo is to fine tune activation states in infected T cells [56]. Nef interferes with TCR proximal signaling to prevent $\mathrm{T}$ cell activation and activation-induced cell death upon antigenic stimulation $[28,30,31,55]$, while at the same time enhancing distal TCR signaling effects to promote proviral expression $[33,57,58]$. Both functions of Nef depend on the interaction of several specific SH3-domain containing host cell proteins with the conserved PxxP motif. For example, the PxxP motif interacts with the SH3- domain of Lck, misrouting the TCR proximal kinase Lck from the plasma membrane to the trans Golgi-network (TGN), altering TCR proximal signaling events [28,30,33,59]. In our HIV replication assay, WT HIV viruses replicated much more efficiently then HIV Nefstop viruses. The requirement of PxxP residues for efficient HIV-1 replication in T-cells is controversial [35,39,44,59]. Lundquist and coworkers did not observe significant differences between the replication-efficiency of WT, AxxA mutant and polyproline deleted viruses [60], while Saksela and co-workers reported that AxxA mutant replicate as deltaNef viruses [35]. In our hands, the replication of the HIV Nef AxxA viruses was significantly attenuated compared to WT viruses. The fact that we use a different proviral backbone and other culture methods for $\mathrm{CD}_{4}^{+}$lymphocyte stimulation may account for the discrepancy between our results and observations from others. Similar to Nef AxxA viruses, VGF $\rightarrow$ AAA mutants replicated much less efficiently than WT HIV viruses. We recently showed that the accumulation of Lck is required for TGN-associated RasErk signaling, which in turn was shown to promote IL-2 production and enhance virus spread [33]. As HIV viruses harboring AxxA or $\mathrm{VGF} \rightarrow \mathrm{AAA}$ mutations in Nef fail to efficiently accumulate Lck [30], this could explain the altered replication kinetics of these mutants. Interestingly, our previous observation that the HIV O8 isolate showed reduced fitness compared to other isolates of the same group might be due to the deletion of the VGF domain [16].

Despite the opposing findings concerning the role of the PxxP motif for replication of HIV, there is general consensus that this domain is required for Nef to enhance the infectivity of HIV virions [61-63]. The mechanism by which Nef enhances viral infectivity remains unclear, but is thought to involve alterations of specific signaling and trafficking events in the producer cell. [9]. We observed that, in line with our other results, VGF $\rightarrow$ AAA mutants were similarly less infective than wild type viruses.

In our study, we only analyzed the importance of the VGF motif in Nef for HIV-1 function. Despite VGF conservation in HIV-1 and SIV $_{\text {cpz }}$; HIV-2 and SIV $_{\text {smm }}$ instead harbor VGV. There are no indications that this change would result in major functional differences, since PxxP dependent functions such as downregulation of MHC-I, accumulation of Lck, deregulation of actin remodeling and association with PAK2 are well conserved between both HIV-1 Nef and HIV-2 Nef [26,28]. Moreover, both phenylalanine and valine are hydrophobic amino acids, conserving the lipophilic character of the entire VGF/V region, and they are not expected to impose a different ultrastructure that would imply different host protein interactions. Rather, the strong conservation of VGF/VGV in HIV/SIV Nef suggests that such a structural motif is required for function of acidic cluster-VGF/V-PxxP motifs in general. 


\section{Conclusions}

Our results extend the previous research performed on the function of the PxxP motif. Based on our and earlier observations from other groups, we propose a new model in which the PxxP motif operates in the context of a larger amphipathic protein surface, encompassing both the acidic cluster and the VGF linker region. Moreover, integrity of the VGF region is required for $\mathrm{SH} 3$ binding and likely for subsequent formation of multi-protein complexes. The fact that a multitude of Nef functions required for enhancement of viral replication and disease progression are dependent on the formation of these protein complexes suggests that the amphipathic region could serve as a prime target for pharmaceutical intervention.

\section{Availability of supporting data}

Nucleotide sequences of isolated HIV-1 and HIV-2 Nef alleles used in this study were submitted to Public sequence repository (Genbank accession numbers JQ 990942 - JQ990960).

\section{Methods}

Viruses and Nef alleles

HIV-1 group $M$ and group $O$ isolates were obtained from the AIDS Research and Reference Reagent Program, while the HIV-2 strains were previously isolated from patients attending the AIDS clinic at the Institute of Tropical Medicine in Antwerp, Belgium, with the approval of the ethical committee. Virus stocks were propagated and expanded in short-term cultures of PBMCs treated with PHA $(1 \mu \mathrm{g} / \mathrm{mL})$ and IL-2 $(10 \mathrm{ng} / \mathrm{mL})$. Genomic DNA was extracted using the Qiagen Blood kit (Qiagen, Venlo, The Netherlands) according to the manufacturer's instructions. Nef alleles from different HIV subtypes were amplified by semi-nested polymerase chain reaction (PCR) with Platinum Pfx DNA polymerase (Invitrogen, Merelbeke, Belgium). Primers recognizing Env and the 3'LTR regions of the HIV genome used for the first round of PCR were forward: 5'-GCACTCAAGGCAAGCTTTATTGAGGC-3' and reverse 5'-CCACATACCTAGAAGAATAAGACAGG-3'. A nested PCR reaction was performed after sequencing and using allelespecific forward and reverse primers covering the complete nef coding sequence and containing restriction sites for BamHI and EcoRI, respectively (see Additional file 1: Table S1A). Site specific mutants of Nef O8, VI1422, B2 and NA-7 in LZRS-IRES-eGFP were generated with PCR using allele specific primers described in Additional file 1: Table S1B and Table S1C.

\section{Plasmid construction}

Nef amplicons were cloned into the LZRS-IRES-eGFP retroviral vector, using BamHI and EcoRI restrictions sites [64]. Plasmids were purified with the Qiaprep Miniprep kit (Qiagen). The integrity of the constructs and the nef genes was confirmed by direct sequencing (ABI, Foster City, California). Alignments of the nef sequences together with selected reference sequences were performed using BioEdit version 5.0.9. Type, group and subtype designation was confirmed by constructing phylogenetic trees by the neighbor joining method using MEGA 4 [65] (see Additional file 1: Figure S1).

Expression constructs for GFP fusion proteins of HIV-1 SF2 Nef, HIV-1 NA-7 Nef and SF2 AxxA were already described and were constructed by cloning PCR fragments of the respective nef genes into pEGFP-N1 (Clontech) under the control of the cytomegalovirus promoter [26,31]. The expression constructs for NA7 Nef VGF $\rightarrow$ AAA and SF2 Nef VGF $\rightarrow$ AAA and NA-7 AxxA were generated by site directed mutagenesis from the wild-type (WT) plasmid (primers Additional file 1: Table S1D).

The proviral constructs used for HIV infectivity and replication assays are derived from the NLENG1-IRES vector [66], which expresses HSA-IRES-Nef cloned in HIV-1 NL43 provirus, as described before [40]. These constructs express from the nef reading frame (together with Nef as two individual proteins) mouse heat-stable antigen as a protein reporter. A BamH1/NgoMIV fragment corresponding to the second exon of tat to the end of nef was cut out from the vector and was ligated into PSUPER [67]. Next, SF2 and NA-7 nef alleles and their specific AxxA and VGF mutants were amplified by PCR using primers containing restriction sites for BstXI and BspEI from the pEGFP-N1 vectors described above. A nef allele, with deleted initiation codon, two in frame stop codons at amino acid positions 4 and 5 and premature stop codons at positions 73 and 74 in the nef ORF, disrupting the nef gene was generated by standard PCR and cloning techniques, similarly to what was described before $\left(\mathrm{Nef}_{\text {stop }}\right)$ [43]. The amplified nef alleles were subsequently cut with BstXI and BspE and ligated into the PSUPER plasmid, swapping it with the WT nef present. NL4.3 AxxA and VGF $\rightarrow$ AAA mutants were generated based on the pSUPER vectors by site directed mutagenesis. Next, BamH1/NgoMIV fragments of newly constructed pSUPER plasmids were ligated into the NL4-3 HSA-IRES-Nef proviral construct. All these final proviral constructs were verified for correct nef allele sequence by direct sequencing.

The vector for the 764 amino acid p59Hck-mCherry fusion protein was constructed by PCR amplifying human Hck cDNA insert from the image clone 4855747 using primers containing BglII (sense) and KpnI (antisense), and inserting it between the BamHI and KpnI sites of the pEBB vector [68], followed by cloning of red fluorescent protein mCherry (Clontech) between the KpnI and EagI sites after the p59Hck insert.

\section{Cell lines, transfection, transduction and flow cytometry} Jurkat TAg [26,29] and Jurkat CD4-CCR5 (Programme EVA Centre for AIDS Reagents, NIBSC, U.K.), are both 
Jurkat E6.1-derived cell lines. The easy to transfect Jurkat TAg cells express the large $T$ antigen of simian virus 40 and were grown in RPMI 1640 medium supplemented with $10 \%$ fetal calf serum (FCS, Hyclone), $100 \mathrm{U} / \mathrm{mL}$ penicillin, and $100 \mathrm{~g} / \mathrm{mL}$ streptomycin (both from Gibco). Jurkat CD4-CCR5 cells were grown in IMDM medium supplemented with $2 \mathrm{mM}$ L-glutamin, 10\% heat-inactivated fetal calf serum (FCS), 293 T cells (American Type Culture Collection (ATCC) Manassas, VA) were maintained in IMDM medium, supplemented with 10\% FCS, $2 \mathrm{mM}$ L-glutamin, $100 \mathrm{U} / \mathrm{mL}$ penicillin, and $100 \mathrm{U} / \mathrm{mL}$ streptomycin.

Mouse antihuman monoclonal antibodies used were: CD4 (clone SK3; $[\mathrm{PE}]$ and $[\mathrm{APC}]$ ), anti-CXCR4, antiMHC-I [PE] (anti-human histocompatibility leukocyte antigens [HLA]-A,-B,-C), all purchased from Becton Dickinson Immunocytometry systems (Beckton Dickinson, Erembodegem, Belgium) and anti-mouse-CD24-APC (HSA, heat stable antigen; BioLegend, San Diego, CA). The cells were analyzed on a FACSCalibur flow cytometer (Becton Dickinson). Forward light scattering, orthogonal scattering, and fluorescence signals were stored and analyzed using the CellQuest software (Becton Dickinson).

Cells were transfected using either calcium-phosphate precipitation (Invitrogen, Merelbeke, Belgium) or JetPei (Polyplus, Sélestat, France) according to the description of the manufacturer. For transduction of suspension cell lines, cells were mixed with retroviral supernatant, preincubated with DOTAP (Roche diagnostics). To increase transduction efficiency, cells were centrifuged (90 minutes, $\left.950 \mathrm{~g}, 32^{\circ} \mathrm{C}\right)[64]$.

\section{Production of retroviral vectors and replication competent HIV}

To produce retroviral vectors, the Phoenix-Amphotropic packaging cell line was transfected with LZRS-IRES-eGFP (control) and LZRS-Nef-IRES-eGFP plasmids using calcium-phosphate precipitation as previously described $[17,64,69]$.

Replication competent HIV was produced by transfecting 293 T cells with the HIV-1 NL4-3 -HSA-IRESNef proviral constructs described above using the JetPEI method. The medium was changed after overnight incubation, and virus was harvested $24 \mathrm{~h}$ later and stored at $-80^{\circ} \mathrm{C}$. The content of viral p24 antigen was quantified using a HIV p24 enzyme-linked immunosorbent assay kit (Innogenetics, Ghent, Belgium).

\section{Immunofluorescence}

Immunofluorescence stainings were performed as previously described [26,28]. Briefly, for staining of F-actin (0.5 $\mathrm{ng} / \mathrm{ml}$ phalloidin-TRITC), cells were fixed for 15 min with 3,7\% paraformaldehyde (PFA)/phosphatebuffered saline (PBS), permeabilized with $0.1 \%$ Triton X100/PBS for $2 \mathrm{~min}$ and subsequently blocked for unspecific binding with $1 \%$ bovine serum albumin (BSA)/PBS for $15 \mathrm{~min}$. Hoechst 33258 was used in a concentration of $1 \mathrm{ng} / \mathrm{ml}$. For staining of phospho-cofilin (p-cofilin) (cell signaling) (1:50), and Lck (Santa Cruz) (1:50) all solutions, dilutions, and wash steps were done with Tris-buffered saline (TBS; $50 \mathrm{mM}$ Tris, $150 \mathrm{mM}$ $\mathrm{NaCl}[\mathrm{pH} 7.5]$ ). Blocking was performed for $30 \mathrm{~min}$, and incubation was performed with the first antibody overnight at $44^{\circ} \mathrm{C}$. Confocal pictures of p-cofilin in Jurkat $\mathrm{T}$ cells were acquired using a Zeiss LSM 510 Axiovert microscope and LSM Meta software. Images were processed using Adobe Photoshop CS3.

\section{In vitro kinase assay}

In vitro kinase assays (IVKA) were essentially performed as described previously [26]. Briefly, Jurkat T lymphocytes were transfected with expression plasmids for Nef. eGFP. After $24 \mathrm{~h}$, cells were lysed in KEB (137 mM $\mathrm{NaCl}, 50 \mathrm{mM}$ Tris/HCl [pH 8], 2 mM EDTA, 0.5\% Nonindet P-40, and protease inhibitors) supplemented with $\mathrm{Na} 3 \mathrm{VO} 4$, and cleared lysates were sampled for Western blot and immunoprecipitated with a rabbit anti-GFP antibody. After intensive washing in KEB, the immunoprecipitates were resuspended in KAB (50 mM HEPES [pH 8], $150 \mathrm{mM} \mathrm{NaCl}, 5 \mathrm{mM}$ EDTA, 0.02\% Triton X$100,10 \mathrm{mM} \mathrm{MgCl} 2)$ containing $10 \mu \mathrm{Ci}$ of $\left[\gamma^{-32} \mathrm{P}\right] \mathrm{ATP}$ per reaction. After incubation for $10 \mathrm{~min}$, samples were washed, and bound proteins were separated by SDSPAGE and subjected to autoradiography. The kinase signal was quantified relative to the SF2 nef allele using Quantity One (Bio-Rad).

\section{Co-immunoprecipitation}

For the Nef-Hck co-immunoprecipitation experiments, $293 \mathrm{~T}$ cells were transfected with expression plasmids for Nef.eGFP and p59Hck (Cherry fusion protein). After $24 \mathrm{~h}$, cells were lysed in KEB $(137 \mathrm{mM} \mathrm{NaCl}, 50 \mathrm{mM}$ Tris/HCl [pH 8], 2 mM EDTA, 0.5\% Nonidet P-40, and protease inhibitors) supplemented with $\mathrm{Na} 3 \mathrm{VO} 4$, and cleared lysates were immunoprecipitated with a GFPTrap (Chromotek, Planegg-Martinsried, Germany). After intensive washing in KEB, the precipitate was incubated in anti-Hck Santa Cruz Biotechnology inc. Santa Cruz, US), diluted 1:1000 in 50\% blocking buffer.

\section{Western blotting}

Nef transduced Jurkat CD4-CCR5 cells sorted for eGFP expression, were lysed in laemmli sample buffer. Equal amounts of protein $(40 \mu \mathrm{g})$ were run on a $12 \%$ precast NuPAGE 4-12\% Bis-Tris polyacrylamide gel (Invitrogen) in reducing conditions and blotted onto a polyvinyldifluoride (PVDF) membrane (Invitrogen). Primary antibodies used were sheep-anti-Nef antiserum (Programme EVA Centre for AIDS Reagents, NIBSC, UK.). Detection 
was done using enhanced chemiluminescence (GE Healthcare, Chalfont St. Gilles, UK). To control that an equal amount of protein was loaded for each sample, blots were also stained for $\beta$-actin (primary anti- $\beta$-actin antibody: clone $\mathrm{C} 4, \mathrm{ICN}$, Aurora, $\mathrm{OH}$, USA).

\section{Infectivity assays}

Virus infectivity was determined using P4R5 HeLa reporter cells (NIH AIDS Reference and Reagent Program) [70]. Briefly, the cells were sown out in 96-well dishes in a volume of $200 \mu \mathrm{I}$ IMDM supplemented with $2 \mathrm{mM} \mathrm{L-gluta-}$ min and 10\% FCS and infected after overnight incubation with virus stocks containing 40 ng of p24 antigen (Innogenetics, Zwijnaarde, Belgium) produced by transiently transfected 293 T cells and centrifuged (90 minutes, $950 \mathrm{~g}, 32^{\circ}$ C). 48 hours postinfection viral infectivity was detected using a beta-galactosidase screen kit from Thermo scientific (Pierce Biotechnology, Rockford, USA) as recommended by the manufacturer. $\beta$-Galactosidase activities were quantified as relative light units per second using an Orion Microplate Luminometer. To calculate percent values, relative light units per second obtained for wild-type HIV-1 NL4-3 HSA-IRES Nef infection were set to $100 \%$.

\section{Viral replication in CD4+ PBL cultures}

To determine the efficiency of HIV-1 replication in peripheral blood $\mathrm{CD}_{4}^{+}$lymphocytes, we isolated $\mathrm{CD}^{+}$cells from buffy coat peripheral blood mononuclear cells (normal blood donors, Red Cross, Ghent, Belgium) by negative selection using paramagnetic beads (MACS; Miltenyi Biotec, Bergish Gladbach, Germany). After isolation, the cells were cultured in RPMI medium supplemented with $2 \mathrm{mM}$ L-glutamin, 10\% heat-inactivated fetal calf serum, phytohemagglutinin $(1 \mu \mathrm{g} / \mathrm{mL}$; Thermo Fisher Scientific, Waltham, USA), $20 \mathrm{ng} / \mathrm{mL}$ IL-2 (Peprotech, Rocky Hill, USA), $100 \mathrm{U} / \mathrm{mL}$ penicillin, and $100 \mathrm{~g} / \mathrm{mL}$ streptomycin. Thereafter, $1 \mathrm{ng}$ of p24 antigen was added to $2,5^{*} 10^{5} \mathrm{PBLs}$, and the culture was spinoculated at 2,300 rpm for 90 minutes at $32^{\circ} \mathrm{C}$. After centrifugation, the supernatant was removed and the cells were further cultured in RPMI supplemented with $20 \mathrm{ng} / \mathrm{mL}$ IL-2. HIV replication was monitored at 1, 3, 5, 7, 9 and 11 days post-infection by measuring the amount of p24 antigen present in the supernatants, using a HIV p24 enzyme-linked immunosorbent assay kit (Innogenetics, Ghent, Belgium). Alternatively, we also determined the expression of the HSA reporter gene at each time point using flow cytometry, as was described before [40].

\section{Statistical analysis}

Data were analysed with non-parametric Mann-Whitney U test, one-tailed (SPSS, version 17; SPSS, Chicago, USA).

\section{Additional File}

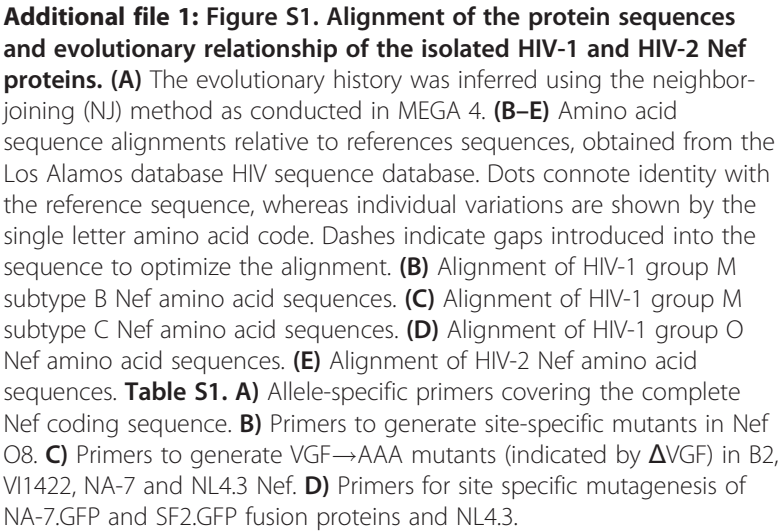

\section{Abbreviations}

HIV: Human immunodeficiency virus; SIV: Simian immunodeficiency virus; AIDS: Acquired immunodeficiency syndrome; TCR: T cell receptor; MHC-I/ II: Major histocompatibility complex class-I/ II; PAK2: P21-activated kinase 2; Lck: Lymphocyte-specific protein tyrosine kinase; TGN: Trans-golgi-network; PBL: Peripheral blood lymphocytes; IL-2: Interleukin-2; PHA: Phytohaemaglutinin A; eGFP: Enhanced green fluorescent protein; PACS-1/2: Phosphofurin acidic cluster- sorting protein-1/2; HSA: Heat stable antigen; AP-1: Adaptor protein 1; DNA: Deoxyribonucleic acid; IRES: Internal ribosomal entry site; FCS: Fetal calf serum; PE: Phycoerythrine; APC: Allophycocyanine; PFA: Paraformaldehyde; IVKA: In vitro kinase assay; PVDF: Polyvinyldifluoride.

\section{Competing interests}

The authors declare that they have no competing interests.

\section{Author's contributions}

PM was responsible for the concept and design of the study, acquisition of most of the data and the writing of the the manuscript; BS was responsible for in vitro kinase and actin ring assays and helped with p-cofilin and Lck assays and critically revised the manuscript; EN gave substantial practical support during acquisition of the data; JV and VI optimized the HIV replication assay, helped with proviral construction and critically revised the manuscript; MG performed in silico modeling, gave substantial scientific input and critically revised the manuscript; KS constructed the p59-HcK expression plasmids and critically revised the manuscript; GV provided the clinical HIV1- and HIV-2 isolates and critically revised the manuscript; KKA performed in vitro culture of primary isolates, is responsible for design of the study and critically revised the article. OF gave substantial scientific input and critically revised the manuscript; BV was responsible for the concept and design of the study, gave substantial scientific input and wrote the manuscript. All authors read and approved the final manuscript.

\section{Acknowledgements}

We would like to thank all the members of our labs for the technical and critical support. We would like to thank the AIDS Research and Reference Reagent Program, Division of AIDS, NIAID, NIH for providing the HIV-1 group $M$ and group $O$ viruses and the HeLa P4R5 cells.The ARP444 sheep antiserum to HIV-1 Nef and ARP094 Jurkat CD4-CCR5 T cell line were obtained from the Programme EVA Centre for AIDS Reagents, NIBSC, U.K., supported by the EC FP6/7 Europrise Network of Excellence, AVIP and NGIN consortia and the Bill and Melinda Gates GHRC-CAVD Project and were donated by Dr. M. Harris, University of Leeds, U.K. and Dr. Q. Sattentau, University of Oxford, U.K., respectively. The authors wish to thank Dr. M. Tremblay (Quebec, Canada) for the gift of the pNL4-3 HSA-IRES-Nef proviral clone and Dr. F. Kirchhoff (Ulm, Germany) for the gift of the Nef Stop clone. This work was supported by SBO CellCoVir grant from the agency for Innovation by Science and Technology (IWT) Flanders, Belgium; HIV-STOP Interuniversity Attraction Poles program of Belgian Science Policy, European Union FP7 Health-2007-2.3.2-1 Collaborative Project iNEF, Ghent University grant BOF11/GOA/013 and grants from the Research Foundation 
- Flanders (FWO) to BV. PJM and JV are PhD. fellows; KKA is a postdoctoral researcher; and BV is a Senior Clinical Investigator of the Fund for Scientific Research-Flanders. VI is a PhD fellow supported by the BOF program of the Ghent University. OTF and BS were supported by the Deutsche Forschungsgemeinschaft (SFB638, TRR83 and GRK1188) and by a fellowship to BS from the Leopoldina/Nationale Akademie der Wissenschaften.

\section{Author details}

${ }^{1}$ Department of Clinical Chemistry, Microbiology, and Immunology, Ghent University, Ghent, (B-9000), Belgium. ²Department of Infectious Diseases, Virology, University Hospital Heidelberg, INF 324, Heidelberg, (D-69120), Germany. ${ }^{3}$ Department of Virology, Haartman Institute, University of Helsinki and Helsinki University Central Hospital, Helsinki, (FIN-00014), Finland. ${ }^{4}$ Max Planck Institute for Molecular Physiology, Dortmund, (D-44227), Germany. ${ }^{5}$ Department of Biomedical Sciences, Virology Unit, Institute of Tropical Medicine, Antwerp, (B-2000), Belgium. ${ }^{6}$ Present Address: Department of Biomedical Sciences, Virology Unit, Institute of Tropical Medicine, Antwerp, Belgium.

\section{Received: 16 December 2011 Accepted: 27 April 2012}

Published: 27 April 2012

\section{References}

1. Daniel MD, Kirchhoff F, Czajak SC, Sehgal PK, Desrosiers RC: Protective effects of a live attenuated SIV vaccine with a deletion in the nef gene. Science 1992, 258:1938-1941.

2. Kestler HW, Ringler DJ, Mori K, Panicali DL, Sehgal PK, Daniel MD, Desrosiers RC: Importance of the Nef Gene for Maintenance of High Virus Loads and for Development of Aids. Cell 1991, 65:651-662.

3. Sawai ET, Hamza MS, Ye M, Shaw KES, Luciw PA: Pathogenic Conversion of Live Attenuated Simian Immunodeficiency Virus Vaccines Is Associated with Expression of Truncated Nef. J Virol 2000, 74:2038-2045.

4. Simard MC, Chrobak P, Kay DG, Hanna Z, Jothy S, Jolicoeur P: Expression of simian immunodeficiency virus nef in immune cells of transgenic mice leads to a severe AIDS-like disease. J Virol 2002, 76:3981-3995.

5. Hanna Z, Kay DG, Rebai N, Guimond A, Jothy S, Jolicoeur P: Nef harbors a major determinant of pathogenicity for an AIDS-like disease induced by HIV-1 in transgenic mice. Cell 1998, 95:163-175.

6. Schwartz O, Marechal V, Le Gall S, Lemonnier F, Heard JM: Endocytosis of major histocompatibility complex class I molecules is induced by the HIV-1 Nef protein. Nat Med 1996, 2:338-342.

7. Landi A, lannucci V, Van Nuffel A, Meuwissen PJ, Verhasselt B: One protein to rule them all: modulation of cell surface receptors and molecules by HIV Nef. Curr HIV Res 2011, 9:496-504

8. Collins KL, Chen BK, Kalams SA, Walker BD, Baltimore D: HIV-1 Nef protein protects infected primary cells against killing by cytotoxic $T$ lymphocytes. Nature 1998, 391:397-401.

9. Vermeire J, Vanbillemont G, Witkowski W, Verhasselt B: The Nef-infectivity enigma: mechanisms of enhanced lentiviral infection. Curr HIV Res 2011, 9:474-489.

10. NJ Arhel F Kirchhoff 2009 Implications of Nef: Host Cell Interactions in Viral Persistence and Progression to AIDSP Spearman EO Freed HIV Interactions with Host Cell Proteins. Current Topics in Microbiology and Immunology Volume 339 Springer-Verlag Berlin Berlin 147175 Arhel NJ, Kirchhoff F: Implications of Nef: Host Cell Interactions in Viral Persistence and Progression to AIDS. In HIV Interactions with Host Cell Proteins. Current Topics in Microbiology and Immunology, Volume Volume 339. Edited by Spearman P, Freed EO. Berlin: Springer-Verlag Berlin; 2009:147-175.

11. Geyer M, Fackler OT, Peterlin BM: Structure-function relationships in HIV-1 Nef. EMBO 2001, 2:580-585.

12. Foster J, Denial S, Temple B, Garcia J: Mechanisms of HIV-1 Nef Function and Intracellular Signaling. J Neuroimm Pharmacol 2011, 6:230-246.

13. show[?twb.4w][?tlsb.09pt]? >Specht A, DeGottardi MQ, Schindler M, Hahn B, Evans DT, Kirchhoff F: Selective downmodulation of HLA-A and -B by Nef alleles from different groups of primate lentiviruses. Virology 2008, 373:229-237.

14. Schindler M, Würfl S, Benaroch P, Greenough TC, Daniels R, Easterbrook P, Brenner M, Münch J, Kirchhoff F: Down-Modulation of Mature Major Histocompatibility Complex Class II and Up-Regulation of Invariant Chain Cell Surface Expression Are Well-Conserved Functions of Human and Simian Immunodeficiency Virus nef Alleles. J Virol 2003, 77:10548-10556.
15. Hrecka K, Swigut T, Schindler M, Kirchhoff F, Skowronski J: Nef Proteins from Diverse Groups of Primate Lentiviruses Downmodulate CXCR4 To Inhibit Migration to the Chemokine Stromal Derived Factor 1. J Virol 2005, 79:10650-10659.

16. Arien KK, Abraha A, Quinones-Mateu ME, Kestens L, Vanham G, Arts EJ: The replicative fitness of primary human immunodeficiency virus type 1 (HIV-1) group M, HIV-1 group O, and HIV-2 isolates. J Virol 2005, 79:8979-8990.

17. Verhasselt B, Naessens E, Verhofstede C, De Smedt M, Schollen S, Kerre T, Vanhecke D, Plum J: Human immunodeficiency virus nef gene expression affects generation and function of human T cells, but not dendritic cells. Blood 1999, 94:2809-2818.

18. Michel N, Ganter K, Venzke S, Bitzegeio J, Fackler OT, Keppler OT: The nef protein of human immunodeficiency virus is a broad-spectrum modulator of chemokine receptor cell surface levels that acts independently of classical motifs for receptor endocytosis and $\mathrm{G}$ alpha(i) signaling. Mol Biol Cell 2006, 17:3578-3590.

19. Venzke S, Michel N, Allespach I, Fackler OT, Keppler OT: Expression of Nef downregulates CXCR4, the major coreceptor of human immunodeficiency virus, from the surfaces of target cells and thereby enhances resistance to superinfection. J Virol 2006, 80:11141-11152.

20. Mangasarian A, Piguet V, Wang J-K, Chen Y-L, Trono D: Nef-Induced CD4 and Major Histocompatibility Complex Class I (MHC-I) Down-Regulation Are Governed by Distinct Determinants: N-Terminal Alpha Helix and Proline Repeat of Nef Selectively Regulate MHC-I Trafficking. J Virol 1999, 73:1964-1973.

21. O'Neill E, Kuo LS, Krisko JF, Tomchick DR, Garcia JV, Foster JL: Dynamic Evolution of the Human Immunodeficiency Virus Type 1 Pathogenic Factor, Nef. J Virol 2006, 80:1311-1320.

22. HIV sequence database, [http:// HIV.lanl.gov], consulted December $15^{\text {th }} 2011$.

23. Breuer S, Schievink SI, Schulte A, Blankenfeldt W, Fackler OT, Geyer M: Molecular Design, Functional Characterization and Structural Basis of a Protein Inhibitor Against the HIV-1 Pathogenicity Factor Nef. PLoS One 2011, 6:e20033.

24. Manninen A, Hiipakka M, Vihinen M, Lu W, Mayer BJ, Saksela K: SH3-Domain Binding Function of HIV-1 Nef Is Required for Association with a PAKRelated Kinase. Virology 1998, 250:273-282.

25. Fackler OT, Luo W, Geyer M, Alberts AS, Peterlin BM: Activation of Vav by Nef Induces Cytoskeletal Rearrangements and Downstream Effector Functions. Mol Cell 1999, 3:729-739.

26. Stolp B, Abraham L, Rudolph JM, Fackler OT: Lentiviral Nef Proteins Utilize PAK2-Mediated Deregulation of Cofilin as a General Strategy To Interfere with Actin Remodeling. J Virol 2010, 84:3935-3948.

27. Sawai ET, Khan IH, Montbriand PM, Peterlin BM, Cheng-Mayer C, Luciw PA: Activation of PAK by HIV and SIV Nef: importance for AIDS in rhesus macaques. Curr Biol 1997, 6:1519-1527.

28. Rudolph JM, Eickel N, Haller C, Schindler M, Fackler OT: Inhibition of T-Cell Receptor-Induced Actin Remodeling and Relocalization of Lck Are Evolutionarily Conserved Activities of Lentiviral Nef Proteins. J Virol 2009, 83:11528-11539.

29. Stolp B, Reichman-Fried M, Abraham L, Pan X, Giese SI, Hannemann S, Goulimari P, Raz E, Grosse R, Fackler OT: HIV-1 Nef Interferes with Host Cell Motility by Deregulation of Cofilin. Cell Host \& Microbe 2009, 6:174-186.

30. Haller C, Rauch S, Fackler OT: HIV-1 Nef Employs Two Distinct Mechanisms to Modulate Lck Subcellular Localization and TCR Induced Actin Remodeling. PLoS One 2007, 2:e1212.

31. Haller C, Rauch S, Michel N, Hannemann S, Lehmann MJ, Keppler OT, Fackler OT: The HIV-1 Pathogenicity Factor Nef Interferes with Maturation of Stimulatory T-lymphocyte Contacts by Modulation of N-Wasp Activity. J Biol Chem 2006, 281:19618-19630.

32. Baugh LL, Garcia JV, Foster JL: Functional Characterization of the Human Immunodeficiency Virus Type 1 Nef Acidic Domain. J Virol 2008, 82:9657-9667.

33. Pan X, Rudolph JM, Abraham L, Habermann A, Haller C, Krijnse-Locker J, Fackler OT: HIV-1 Nef compensates for disorganization of the immunological synapse by inducing TGN-associated Lck signaling. Blood 2011, 119: 786-797.

34. Thoulouze MI, Sol-Foulon N, Blanchet F, Dautry-Varsat A, Schwartz O, Alcover A: Human Immunodeficiency Virus Type-1 Infection Impairs the Formation of the Immunological Synapse. Immunity 2006, 24:547-561.

35. Saksela K, Cheng G, Baltimore D: Proline-rich (PxxP) motifs in HIV-1 Nef bind to $\mathrm{SH} 3$ domains of a subset of Src kinases and are required for the 
enhanced growth of Nef + viruses but not for down-regulation of CD4. EMBO 1995, 14:484-491.

36. Hammes SR, Dixon EP, Malim MH, Cullen BR, Greene WC: Nef protein of human immunodeficiency virus type 1: evidence against its role as a transcriptional inhibitor. Proc Nat Acad Sci 1989, 86:9549-9553.

37. Kim S, Ikeuchi K, Byrn R, Groopman J, Baltimore D: Lack of a negative influence on viral growth by the nef gene of human immunodeficiency virus type 1. Proc Nat Acad Sci 1989, 86:9544-9548.

38. Spina CA, Kwoh TJ, Chowers MY, Guatelli JC, Richman DD: The importance of nef in the induction of human immunodeficiency virus type 1 replication from primary quiescent CD4 lymphocytes. J Exp Med 1994, 179:115-123.

39. Homann S, Tibroni N, Baumann I, Sertel S, Keppler O, Fackler O: Determinants in HIV-1 Nef for enhancement of virus replication and depletion of CD4+ T lymphocytes in human lymphoid tissue ex vivo. Retrovirology 2009, 6:6.

40. Imbeault MI, Lodge R, Ouellet M, Tremblay MJ: Efficient magnetic beadbased separation of HIV-1-infected cells using an improved reporter virus system reveals that p53 up-regulation occurs exclusively in the virus-expressing cell population. Virology 2009, 393:160-167.

41. Fackler OT, Moris A, Tibroni N, Giese SI, Glass Br, Schwartz O, Krausslich H-G Functional characterization of HIV-1 Nef mutants in the context of viral infection. Virology 2006, 351:322-339.

42. Rücker E, Münch J, Wildum S, Brenner M, Eisemann J, Margolis L, Kirchhoff F: A Naturally Occurring Variation in the Proline-Rich Region Does Not Attenuate Human Immunodeficiency Virus Type 1 Nef Function. J Virol 2004, 78:10197-10201

43. Münch J, Rajan D, Schindler M, Specht A, Rücker E, Novembre FJ, Nerrienet E, Müller-Trutwin MC, Peeters M, Hahn BH, Kirchhoff F: Nef-Mediated Enhancement of Virion Infectivity and Stimulation of Viral Replication Are Fundamental Properties of Primate Lentiviruses. J Virol 2007, 81:13852-13864

44. Fackler OT, Wolf D, Weber HO, Laffert B, D'Aloja P, Schuler-Thurner B, Geffin $R$, Saksela K, Geyer M, Peterlin BM, et al: A natural variability in the prolinerich motif of Nef modulates HIV-1 replication in primary T cells. Curr Biol 2001, 11:1294-1299.

45. Brown A, Moghaddam S, Kawano T, Cheng-Mayer C: Multiple human immunodeficiency virus type 1 Nef functions contribute to efficient replication in primary human macrophages. J Gen Virol 2004, 85:1463-1469.

46. Geyer $M$, Peterlin BM: Domain assembly, surface accessibility and sequence conservation in full length HIV-1 Nef. FEBS 2001, 496:91-95.

47. Saksela K: Interactions of HIV/SIV pathogenicity factor nef with SH3 domain containing host cell proteins. Curr HIV Res 2011, 9: S31-S42.

48. Greenberg ME, lafrate AJ, Skowronski J: The SH3 domain-binding surface and an acidic motif in HIV-1 Nef regulate trafficking of class I MHC complexes. EMBO 1998, 17:2777-2789.

49. Mangino G, Percario ZA, Fiorucci G, Vaccari G, Acconcia F, Chiarabelli C, Leone S, Noto A, Horenkamp FA, Manrique S, et al: HIV-1 Nef Induces Proinflammatory State in Macrophages through Its Acidic Cluster Domain: Involvement of TNF Alpha Receptor Associated Factor 2. PLoS One 2011, 6:e22982

50. Blagoveshchenskaya AD, Thomas L, Feliciangeli SF, Hung C-H, Thomas G: HIV-1 Nef Downregulates MHC-I by a PACS-1- and PI3K-Regulated ARF6 Endocytic Pathway. Cell 2002, 111:853-866.

51. Atkins KM, Thomas L, Youker RT, Harriff MJ, Pissani F, You H, Thomas G: HIV-1 Nef Binds PACS-2 to Assemble a Multikinase Cascade That Triggers Major Histocompatibility Complex Class I (MHC-1) Down-regulation. J Biol Chem 2008, 283:11772-11784.

52. Schaefer MR, Wonderlich ER, Roeth JF, Leonard JA, Collins KL: HIV-1 Nef Targets MHC-I and CD4 for Degradation Via a Final Common beta-COPDependent Pathway in T Cells. PLoS Pathog 2008, 4:e1000131.

53. Singh RK, Lau D, Noviello CM, Ghosh P, Guatelli JC: An MHC-I Cytoplasmic Domain/HIV-1 Nef Fusion Protein Binds Directly to the mu Subunit of the AP-1 Endosomal Coat Complex. PLoS One 2009, 4:e8364.

54. Ball $\mathrm{L}$, Jarchau T, Oschkinat $\mathrm{H}$, Walter $\mathrm{U}: \mathrm{EVH} 1$ domains: structure, function and interactions. FEBS Lett 2002, 513(1):45-52

55. Shelton MN, Huang MB, Ali SA, Powell MD, Bond VC: Secretion modification region-derived peptide disrupts HIV-1 Nef's interaction with mortalin and blocks virus and nef exsosome release. J Virol 2012, 86 (1):406-19.

56. Stevenson M: HIV-1 pathogenesis. Nat Med 2003, 9:853-860.
57. Arhel N, Lehmann M, Clauss K, Nienhaus GU, Piguet V, Kirchhoff F: The inability to disrupt the immunological synapse between infected human T cells and APCs distinguishes HIV-1 from most other primate lentiviruses. J Clin Invest 2009, 119:2965-2975.

58. Simmons A, Aluvihare V, McMichael A: Nef Triggers a Transcriptional Program in T Cells Imitating Single-Signal T Cell Activation and Inducing HIV Virulence Mediators. Immunity 2001, 14:763-777.

59. Fenard D, Yonemoto W, de Noronha C, Cavrois M, Williams SA, Greene WC: Nef Is Physically Recruited into the Immunological Synapse and Potentiates T Cell Activation Early after TCR Engagement. J Immunol 2005, 175:6050-6057.

60. Lundquist CA, Tobiume M, Zhou J, Unutmaz D, Aiken C: Nef-Mediated Downregulation of CD4 Enhances Human Immunodeficiency Virus Type 1 Replication in Primary T Lymphocytes. J Virol 2002, 76:4625-4633.

61. Madrid R, Janvier K, Hitchin D, Day J, Coleman S, Noviello C, Bouchet J, Benmerah A, Guatelli J, Benichou S: Nef-induced Alteration of the Early/ Recycling Endosomal Compartment Correlates with Enhancement of HIV-1 Infectivity. J Biol Chem 2005, 280:5032-5044.

62. Goldsmith MA, Warmerdam MT, Atchison RE, Miller MD, Greene WC Dissociation of the CD4 downregulation and viral infectivity enhancement functions of human immunodeficiency virus type 1 Nef. J Virol 1995, 69:4112-4121.

63. Chutiwitoonchai N, Hiyoshi M, Mwimanzi P, Ueno T, Adachi A, Ode H, Sato H, Fackler OT, Okada S, Suzu S: The Identification of a Small Molecule Compound That Reduces HIV-1 Nef-Mediated Viral Infectivity Enhancement. PLoS One 2011, 6:e27696.

64. Stove V, Naessens E, Stove C, Swigut T, Plum J, Verhasselt B: Signaling but not trafficking function of HIV-1 protein Nef is essential for Nef-induced defects in human intrathymic T-cell development. Blood 2003, 102:2925-2932.

65. Tamura K, Dudley J, Nei M, Kumar S: MEGA4: Molecular evolutionary genetics analysis (MEGA) software version 4.0. Mol Biol Evol 2007, 24:1596-1599.

66. Levy DN, Aldrovandi GM, Kutsch O, Shaw GM: Dynamics of HIV-1 recombination in its natural target cells. Proc Nat Acad Sci 2004, 101:4204-4209.

67. Brummelkamp TR, Bernards R, Agami R: A system for stable expression of short interfering RNAs in mammalian cells. Science 2002, 296:550-553.

68. Tanaka M, Gupta R, Mayer BJ: Differential inhibition of signaling pathways by dominant-negative SH2/SH3 adapter proteins. Mol Cell Biol 1995, 15:6829-6837.

69. Smits K, lannucci V, Stove V, Van Hauwe P, Naessens EL, Meuwissen PJ, Arien KK, Bentahir M, Plum J, Verhasselt B: Rho GTPase Cdc42 is essential for human T-cell development. Haematologica 2010, 95:367-375.

70. Deng H, Liu R, Ellmeier W, Choe S, Unutmaz D, Burkhart M, Marzio PD, Marmon S, Sutton RE, Hill CM, et al: Identification of a major co-receptor for primary isolates of HIV-1. Nature 1996, 381:661-666.

doi:10.1186/1742-4690-9-34

Cite this article as: Meuwissen et al: Identification of a highly conserved valine-glycine-phenylalanine amino acid triplet required for HIV-1 Nef function. Retrovirology 2012 9:34.

\section{Submit your next manuscript to BioMed Central and take full advantage of:}

- Convenient online submission

- Thorough peer review

- No space constraints or color figure charges

- Immediate publication on acceptance

- Inclusion in PubMed, CAS, Scopus and Google Scholar

- Research which is freely available for redistribution 